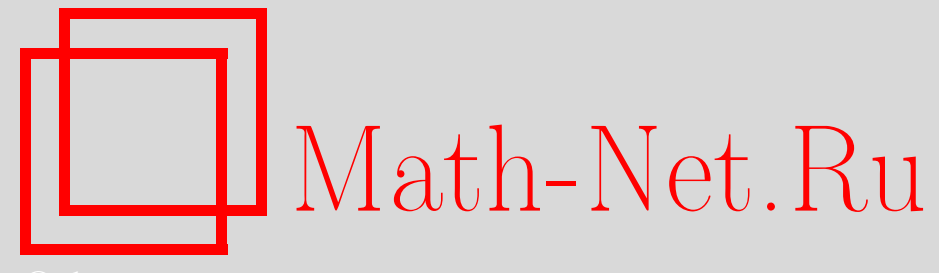

P. Di Tella, H.-J. Engelbert, The predictable representation property of compensated-covariation stable families of martingales, Теория вероятн. и ее примен., 2015, том 60, выпуск 1, 99-130

DOI: https://doi.org/10.4213/tvp4607

Использование Общероссийского математического портала Math-Net.Ru подразумевает, что вы прочитали и согласны с пользовательским соглашением http://www . mathnet.ru/rus/agreement

Параметры загрузки:

IP : 18.207 .199 .55

26 апреля 2023 г., $10: 28: 20$

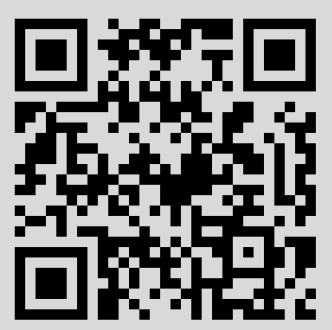




\title{
THE PREDICTABLE REPRESENTATION PROPERTY OF COMPENSATED-COVARIATION STABLE FAMILIES OF MARTINGALES ${ }^{1)}$
}

\begin{abstract}
Изучается свойство предсказуемого представления некоторых семейств квадратично интегрируемых мартингалов, которые мы называем устойчивыми семействами с компенсированными ковариациями. Определение свойства предсказуемого представления дается с помощью устойчивых подпространств. Основной результат состоит в том, что устойчивые семейства с компенсированными ковариациями, удовлетворяющие некоторым дополнительным условиям, обладают свойством предсказуемого представления. В качестве первых примеров мы рассматриваем непрерывные гауссовские семейства мартингалов и независимые семейства компенсированных пуассоновских процессов. Затем мы применяем наш результат к процессам Леви. Мы строим семейства мартингалов относительно фильтрации Леви, обладающие свойством предсказуемого представления. Мы рассматриваем несколько примеров, в том числе мартингалы Тойгельса.
\end{abstract}

Ключевые слова и фразы: квадратично интегрируемые мартингалы, ортогональные мартингалы, устойчивые подпространства, свойство предсказуемого представления, процессы Леви, мартингалы Тойгельса.

1. Introduction. In this paper we investigate the predictable representation property (PRP) for so-called compensated-covariation stable families of martingales in the Hilbert space of square integrable martingales.

A local martingale $(X, \mathbb{F})$ is said to possess the PRP with respect to the filtration $\mathbb{F}$ if every $\mathbb{F}$-local martingale can be represented as stochastic integral with respect to it. It is well known that the Wiener process $W$ has the PRP with respect to the augmented filtration $\mathbb{F}^{W}$ generated by $W$. A seminal paper for the study of the PRP is [11], where the multiple integral for the Wiener process was defined. The first articles in which the PRP for the Wiener process was proved are [20] and [2]. In [16] and [17] analogous results were obtained for the compensated Poisson process.

\footnotetext{
${ }^{*}$ Humboldt-Universität zu Berlin, Berlin, Germany; e-mail: ditellap@math.hu-berlin.de

**Friedrich-Schiller-Universität Jena, Jena, Germany; e-mail: hans-juergen.engelbert @uni-jena.de

1) This work was financially supported by the European Community's FP 7 Program under contract PITN-GA-2008-213841, Marie Curie ITN «Controlled Systems».
} 
The Wiener process and the compensated Poisson process are not the unique local martingales possessing the PRP with respect to their generated augmented filtration. In a series of papers, among others [3], [1], [5], and [7], representation results were obtained for local martingales with respect to the filtration generated by a certain kind of jump processes. In [8] conditions of probabilistic type were given for general continuous local martingales $X$ to have the PRP with respect to the augmented filtration $\mathbb{F}^{X}$ generated by $X$. In [4] the problem of the multiplicity of a filtration was considered.

The formulation of the PRP given above is appropriate to deal with only one martingale and all the mentioned results were obtained either in this case or for finitely many orthogonal martingales. However, the formulation of the PRP for finite families of local martingales is too restrictive and only special processes fulfil it. For example, in the case of a single local martingale, the Wiener process and the compensated Poisson process are the unique Lévy processes having the PRP. Therefore, it is reasonable to extend the definition of the PRP to arbitrary (in particular, infinite) families of local martingales to hope to find a larger class of martingales satisfying it. This extension makes use of the theory of stable subspaces presented in [15] and [13].

Let $\mathscr{X}$ be a family of locally square-integrable local martingales on the complete probability space $\left(\Omega, \mathscr{F}_{\infty}^{\mathscr{X}}, \mathbb{P}\right)$ and with respect to the filtration $\mathbb{F}_{+}^{\mathscr{X}}$, where $\mathscr{F}_{\infty}^{\mathscr{X}}$ denotes the smallest complete $\sigma$-algebra such that $\mathscr{X}$ is a measurable family, while $\mathbb{F}_{+}^{\mathscr{X}}$ is the smallest filtration satisfying the usual conditions such that $\mathscr{X}$ is a family of adapted processes. We look for sufficient conditions on the family $\mathscr{X}$ to ensure that it possesses the PRP with respect to $\mathbb{F}_{+}^{\mathscr{X}}$. Given an arbitrary index set $I$ and the family $\mathscr{X}:=\left\{X^{\alpha}, \alpha \in I\right\}$ of locally square integrable local martingales in the parametric form, we call the process $X^{\alpha, \beta}:=\left[X^{\alpha}, X^{\beta}\right]-\left\langle X^{\alpha}, X^{\beta}\right\rangle$ the compensated-covariation process (of $X^{\alpha}$ and $X^{\beta}$ ), $\alpha, \beta \in I$. Obviously $X^{\alpha, \beta}$ is a local martingale. We say that the family $\mathscr{X}$ is compensated-covariation stable if $X^{\alpha, \beta}$ belongs to $\mathscr{X}$, for every $\alpha, \beta \in I$. The main result of this paper is that if $\mathscr{X}$ is a compensated-covariation stable family of true locally square integrable $\mathbb{F}_{+}^{\mathscr{X}}$ martingales such that $\left\langle X^{\alpha}, X^{\beta}\right\rangle$ is deterministic, for every $\alpha, \beta \in I$, and if the system of polynomials generated by $\mathscr{X}$ is dense in $L^{2}\left(\Omega, \mathscr{F}_{\infty}^{\mathscr{X}}, \mathbb{P}\right)$, then $\mathscr{X}$ possesses the PRP relative to $\mathbb{F}_{+}^{\mathscr{X}}$.

Section 2 is a brief recall of the general theory which we shall need. We introduce stochastic integrals with respect to locally square integrable local martingales, the theory of stable subspaces, and the general formulation of the PRP.

In Section 3 we discuss compensated-covariation stable families of locally square integrable local martingales and we give sufficient conditions for such families to possess the PRP with respect to $\mathbb{F}_{+} \mathscr{X}$.

In Section 4 we apply the theory developed in Section 3 to Lévy processes. More precisely we consider a Lévy process $\left(L, \mathbb{F}^{L}\right)$ with Lévy measure 
$\nu$, where $\mathbb{F}^{L}$ denotes the completed filtration generated by $L$, and in a first step we construct compensated-covariation stable families of $\mathbb{F}^{L}$-martingales with the PRP relative to $\mathbb{F}^{L}$. As a consequence we give a characterization of the PRP for certain systems of $\mathbb{F}^{L}$-martingales for Lévy processes. We conclude discussing some examples of martingales possessing the PRP with respect to $\mathbb{F}^{L}$.

\section{Preliminaries.}

2.1. Basic definitions and notations. In this subsection we recall some basic definitions and notation that we shall need in the sequel. We assume that a complete probability space $(\Omega, \mathscr{F}, \mathbb{P})$ is given and we fix a filtration $\mathbb{F}$ which satisfies the usual conditions. We shall always consider real-valued stochastic processes.

We say that a process $X=\left(X_{t}\right)_{t \geqslant 0}$ admits a terminal variable $X_{\infty}$ if $X_{t}$ converges a.s. to a limit $X_{\infty}$ as $t \uparrow \infty$. We stress that the random variable $X_{\infty}$ takes values in $\mathbb{R} \cup\{ \pm \infty\}$.

Let $X=\left(X_{t}\right)_{t \geqslant 0}$ be a càdlàg process. We define the process $X_{-}=$ $\left(X_{t-}\right)_{t \geqslant 0}$ by setting $X_{0-}:=X_{0}$ and $X_{t-}:=\lim _{s \uparrow t} X_{s}$ if $t>0$. The process $\Delta X=\left(\Delta X_{t}\right)_{t \geqslant 0}, \Delta X_{t}:=X_{t}-X_{t-}, t \geqslant 0$, is called the jump process of $X$. Note that $\Delta X_{0}=0$.

We denote by $\mathscr{M}=\mathscr{M}(\mathbb{F})$ the class of uniformly integrable $\mathbb{F}$ martingales with initial value equal to zero. Here martingales are assumed to be càdlàg processes. The class $\mathscr{M}_{\text {loc }}$ is introduced from $\mathscr{M}$ by localization and is the class of $\mathbb{F}$-local martingales with initial value equal to zero. For $X \in \mathscr{M}_{\text {loc }}$ we denote by $X^{c}$ and $X^{d}$ the continuous and the purely discontinuous part of $X$, respectively (cf. [14, Theorem I.4.18]).

If $X$ and $Y$ belong to $\mathscr{M}_{\text {loc }}$, we say that they are orthogonal and write $X \perp Y$ if their product $X Y$ belongs to $\mathscr{M}_{\text {loc }}$. If $\mathscr{X} \subseteq \mathscr{M}_{\text {loc }}$ we say that $X \in \mathscr{M}_{\text {loc }}$ is orthogonal to $\mathscr{X}$ if $X \perp Y$ for every $Y \in \mathscr{X}$.

By $\mathscr{V}^{+}$(respectively, $\mathscr{V}$ ) we denote the set of adapted càdlàg processes starting at zero with increasing paths (respectively, paths of finite variation on compact intervals). If $A$ belongs to $\mathscr{V}^{+}$(respectively, $\left.\mathscr{V}\right)$, then we say that $A$ is an increasing process (respectively, a process of finite variation). For $A \in \mathscr{V}$, by $\operatorname{Var}(A)=\left(\operatorname{Var}(A)_{t}\right)_{t \geqslant 0}$ we denote the variation process of $A$. We denote by $\mathscr{A}^{+}$the set of increasing processes with integrable terminal variable. By $\mathscr{A}$ we denote the set of processes $A \in \mathscr{V}$ such that $\operatorname{Var}(A) \in \mathscr{A}^{+}$. The class $\mathscr{A}_{\text {loc }}^{+}$(respectively, $\mathscr{A}_{\text {loc }}$ ) is introduced from $\mathscr{A}^{+}$ (respectively, $\mathscr{A}$ ) by localization.

For any $A \in \mathscr{V}$ and measurable process $H$ we shall make use of the Riemann-Stieltjes integral of $H$ with respect to $A$, denoted by $H \cdot A_{t}(\omega):=$ $\int_{0}^{t} H_{s}(\omega) \mathrm{d} A_{s}(\omega)$ for $t \geqslant 0$ and by $H \cdot A=\left(H \cdot A_{t}\right)_{t \geqslant 0}$ for the integral process (cf. [14, Chap. I, §3a]). We stress that if $A \in \mathscr{V}$ (respectively, $A \in \mathscr{V}^{+}$) and $H$ is a measurable process (respectively, a nonnegative measurable process) 
such that $H \cdot A_{t}(\omega)$ is finite-valued for every $t \geqslant 0$ and $\omega \in \Omega$, then $H \cdot A \in \mathscr{V}$ (respectively, $H \cdot A \in \mathscr{V}^{+}$).

We consider $A \in \mathscr{V}$ and two general measurable processes $H, K$ such that the processes $K \cdot A$ and $H \cdot(K \cdot A)$ are well defined and belong to $\mathscr{V}$. Under these assumptions it is straightforward to prove that the process $(H K) \cdot A$ belongs to $\mathscr{V}$ and that the following identity holds:

$$
H \cdot(K \cdot A)=(H K) \cdot A .
$$

We say that a martingale $X \in \mathscr{M}$ is square integrable if $\sup _{t \geqslant 0}\left(X_{t}\right)^{2}$ is integrable. We denote by $\mathscr{H}^{2}$ the set of square integrable martingales. For any $X$ from $\mathscr{H}^{2}$ we introduce the norm $\|X\|_{\mathscr{H}^{2}}:=\left\|X_{\infty}\right\|_{2}$, where $\|\cdot\|_{2}$ denotes the $L^{2}(\mathbb{P}):=L^{2}(\Omega, \mathscr{F}, \mathbb{P})$-norm, and for $X, Y \in \mathscr{H}^{2}$, we put $(X, Y)_{\mathscr{H}^{2}}:=\mathbb{E}\left[X_{\infty} Y_{\infty}\right]$, which defines a scalar product on $\mathscr{H}^{2}$. We can identify $\left(\mathscr{H}^{2},\|\cdot\|_{\mathscr{H}^{2}}\right)$ with the Hilbert space $\left(L^{2}(\mathbb{P}),\|\cdot\|_{2}\right)$.

The space $\mathscr{H}_{\text {loc }}^{2}$ is defined from $\mathscr{H}^{2}$ by localization and is the space of locally square integrable local martingales. If $X$ and $Y$ belong to $\mathscr{H}_{\mathrm{loc}}^{2}$, there exists a unique predictable process belonging to $\mathscr{V}$, which we denote by $\langle X, Y\rangle$ and call the point brackets of $X$ and $Y$, characterized by $X Y-$ $\langle X, Y\rangle \in \mathscr{M}_{\text {loc }}$ (cf. [14, Theorem I.4.2]). For $X, Y \in \mathscr{H}_{\text {loc }}^{2}$, then $X \perp Y$ if and only if $\langle X, Y\rangle=0$. We also recall that, if $X, Y \in \mathscr{H}^{2}$, then $\langle X, Y\rangle \in \mathscr{A}$, the process $X Y-\langle X, Y\rangle$ belongs to $\mathscr{M}$ and $\mathbb{E}\left[X_{\infty} Y_{\infty}\right]=\mathbb{E}\left[\langle X, Y\rangle_{\infty}\right]$.

Let $(X, \mathbb{F})$ be a semimartingale with decomposition $X=X_{0}+M+A$, where $M \in \mathscr{M}_{\text {loc }}, A \in \mathscr{V}$, and $X_{0}$ is $\mathscr{F}_{0}$-measurable. The continuous martingale part of $X$, denoted by $X^{c}$, is defined by $X^{c}:=M^{c}$. Note that $X^{c}$ does not depend on the semimartingale decomposition (cf. [14, Proposition I.4.27]). With two semimartingales $X$ and $Y$, we can associate the process $[X, Y]$, called covariation of $X$ and $Y$, defining

$$
[X, Y]_{t}:=\left\langle X^{c}, Y^{c}\right\rangle_{t}+\sum_{0<s \leqslant t} \Delta X_{s} \Delta Y_{s}, \quad t \geqslant 0 .
$$

It is well known that the process $[X, Y]$ belongs to $\mathscr{V}$ (cf. [13, Theorem 2.30]) and, for every stopping time $T$, we have $[X, Y]^{T}=\left[X^{T}, Y\right]=\left[X^{T}, Y^{T}\right]$. If $X, Y \in \mathscr{H}_{\mathrm{loc}}^{2}$, then $[X, Y],\langle X, Y\rangle \in \mathscr{A}_{\text {loc }}$ and $\langle X, Y\rangle$ is the compensator of $[X, Y]$, i.e., $\langle X, Y\rangle$ is the unique predictable process from $\mathscr{A}_{\text {loc }}$ such that $[X, Y]-\langle X, Y\rangle \in \mathscr{M}_{\mathrm{loc}}$.

In [14, Proposition I.4.49a] it is stated that if $X$ is a semimartingale and $A$ belongs to $\mathscr{V}$, then the process $\Delta X \cdot A$ belongs to $\mathscr{V}$ and the covariation of $X$ and $A$ can be written as an integral with respect to $A$ as follows:

$$
[X, A]=\Delta X \cdot A .
$$

The following lemma is immediately deduced from (3) and (1) by induction. 
Lemma 2.1. If $X^{1}, \ldots, X^{m}$ are semimartingales, $m \geqslant 1$, and $A$ belongs to $\mathscr{V}$, then the integral $\left(\prod_{i=1}^{m} \Delta X^{i}\right) \cdot A$ belongs to $\mathscr{V}$ as well.

We shall use the stochastic integral with respect to locally square integrable local martingales $X \in \mathscr{H}_{\text {loc }}^{2}$. For $X \in \mathscr{H}_{\text {loc }}^{2}$, the space of square integrable predictable integrands is denoted by $\mathrm{L}^{2}(X):=\left\{H\right.$ predictable: $\mathbb{E}\left[H^{2}\right.$. $\left.\left.\langle X, X\rangle_{\infty}\right]<+\infty\right\} ; \mathrm{L}_{\text {loc }}^{2}(X)$ is the space of predictable processes $H$ such that $H^{2} \cdot\langle X, X\rangle \in \mathscr{A}_{\text {loc }}^{+}$. Notice that any locally bounded predictable $H$ belongs to $\mathrm{L}_{\text {loc }}^{2}(X)$. If $H \in \mathrm{L}_{\text {loc }}^{2}(X)$, by $H \cdot X$ we denote the stochastic integral of $H$ with respect to $X$. Let $Z \in \mathscr{H}_{\mathrm{loc}}^{2}$. Then $Z=H \cdot X$ if and only if $\langle Z, Y\rangle=H \cdot\langle X, Y\rangle$ for every $Y \in \mathscr{H}_{\text {loc }}^{2}$. Furthermore, $H \cdot X \in \mathscr{H}^{2}$ if and only if $H \in \mathrm{L}^{2}(X)$. The notation $H \cdot X$ is not ambiguous with the one introduced for the stochastic integral with respect to a process of finite variation: If $X \in \mathscr{H}_{\text {loc }}^{2} \cap \mathscr{V}$, then $H \cdot X$ coincides with the Riemann-Stieltjes integral (cf. [13, Remark 2.47]). For any stopping time $T, X \in \mathscr{H}_{\mathrm{loc}}^{2}$, and $H \in \mathrm{L}^{2}(X)$, we have

$$
(H \cdot X)^{T}=\left(H 1_{[0, T]}\right) \cdot X=H \cdot X^{T} .
$$

Recalling that $(H \cdot X)^{c}=H \cdot X^{c}$ and $\Delta(H \cdot X)=H \Delta X$, it is immediate to verify that for $X, Y \in \mathscr{H}_{\mathrm{loc}}^{2}, H \in \mathrm{L}^{2}(X)$, and $K \in \mathrm{L}^{2}(Y)$, the stochastic integral fulfils the relation (also valid for $X$ and $Y$ semimartingales and $H$ and $K$ locally bounded and predictable; cf. [14, I.4.54])

$$
[H \cdot X, K \cdot Y]=H K \cdot[X, Y]
$$

2.2. Stable subspaces and predictable representation property. In this subsection we give the formulation of the predictable representation property $(\mathrm{PRP})$ for an arbitrary subset $\mathscr{X}$ of $\mathscr{H}_{\text {loc }}^{2}$. For this aim we use the notion of the stable subspace generated by $\mathscr{X}$. We refer to [13, Chapters IV and XI].

Let $\mathscr{H} \subseteq \mathscr{H}^{2}$ be a linear space. We say that $\mathscr{H}$ is a stable subspace of $\mathscr{H}^{2}$ if it is closed in the Hilbert space $\left(\mathscr{H}^{2},\|\cdot\| \mathscr{H}^{2}\right)$ and stable under stopping, i.e., $X^{T} \in \mathscr{H}$ for every stopping time $T$ and $X \in \mathscr{H}$. A linear subspace $\mathscr{H} \subseteq \mathscr{H}^{2}$ is a stable subspace of $\mathscr{H}^{2}$ if and only if it is closed in $\left(\mathscr{H}^{2},\|\cdot\| \mathscr{H}^{2}\right)$ and stable under stochastic integration, i.e., $H \cdot X \in \mathscr{H}$ for every $H \in \mathrm{L}^{2}(X)$ and $X \in \mathscr{H}$ (cf. [13, Proposition 4.3]).

For any family $\mathscr{X} \subseteq \mathscr{H}_{\text {loc }}^{2}$, the stable subspace generated by $\mathscr{X}$ in $\mathscr{H}^{2}$ is denoted by $\mathscr{L}^{2}(\mathscr{X})$. If $\mathscr{X} \subseteq \mathscr{H}^{2}$, then $\mathscr{L}^{2}(\mathscr{X})$ is defined as the smallest stable subspace of $\mathscr{H}^{2}$ containing $\mathscr{X}$. If $\mathscr{X} \subseteq \mathscr{H}_{\text {loc }}^{2}$, then $\mathscr{L}^{2}(\mathscr{X})$ is defined as the stable subspace generated in $\mathscr{H}^{2}$ by the subfamily $\left\{H \cdot X: H \in \mathrm{L}^{2}(X), X \in \mathscr{X}\right\}$ of $\mathscr{H}^{2}$ and coincides with the stable subspace of $\mathscr{H}^{2}$ generated by the system $\left\{X^{T}: X \in \mathscr{X}, T\right.$ stopping time such that $\left.X^{T} \in \mathscr{H}^{2}\right\}$.

The following result describes $\mathscr{L}^{2}(\mathscr{X})$ for $\mathscr{X} \subseteq \mathscr{H}_{\mathrm{loc}}^{2}$. We refer to $[13$, Theorem 4.6 and Proposition 4.7], and use the following notation: For a class 
$\mathscr{C}$ of real-valued functions or processes, we denote by $\operatorname{Span}(\mathscr{C})$ the linear hull of $\mathscr{C}$.

Theorem 2.1. (i) If $X \in \mathscr{H}_{\mathrm{loc}}^{2}$, then $\mathscr{L}^{2}(X)=\left\{H \cdot X, H \in \mathrm{L}^{2}(X)\right\}$.

(ii) If $\mathscr{X} \subseteq \mathscr{H}_{\text {loc }}^{2}$, then $\mathscr{L}^{2}(\mathscr{X})$ is the closure of $\operatorname{Span}\left(\bigcup_{X \in \mathscr{X}} \mathscr{L}^{2}(X)\right)$ in $\mathscr{H}^{2}$ with respect to the norm $\|\cdot\|_{\mathscr{H}^{2}}$.

If $X$ and $Y$ are orthogonal local martingales belonging to $\mathscr{H}_{\mathrm{loc}}^{2}$, then the stable subspaces $\mathscr{L}^{2}(X)$ and $\mathscr{L}^{2}(Y)$ are orthogonal closed subspaces of $\left(\mathscr{H}^{2},\|\cdot\|_{\mathscr{H}^{2}}\right)$. Indeed, Theorem 2.1 (i) yields that for every $Z^{1} \in \mathscr{L}^{2}(X)$ and $Z^{2} \in \mathscr{L}^{2}(Y)$ there exist processes $H \in \mathrm{L}^{2}(X)$ and $K \in \mathrm{L}^{2}(Y)$ such that $Z^{1}=H \cdot X$ and $Z^{2}=K \cdot Y$. Moreover it follows $\left\langle Z^{1}, Z^{2}\right\rangle=\langle H \cdot X, K \cdot Y\rangle=$ $H K \cdot\langle X, Y\rangle=0$. Therefore, $Z^{1} \perp Z^{2}$ and $Z^{1} Z^{2} \in \mathscr{M}$. This means that $\mathbb{E}\left[Z_{\infty}^{1} Z_{\infty}^{2}\right]=\mathbb{E}\left[\left\langle Z^{1}, Z^{2}\right\rangle_{\infty}\right]=0$, i.e., $Z^{1}$ and $Z^{2}$ are orthogonal in the Hilbert space $\left(\mathscr{H}^{2},\|\cdot\|_{\mathscr{H}^{2}}\right)$.

If $\mathscr{X} \subseteq \mathscr{H}_{\text {loc }}^{2}$ is an orthogonal and countable family, say $\mathscr{X}:=\left\{X^{n}\right.$, $n \geqslant 1\}$, then the structure of the stable subspace generated by $\mathscr{X}$ in $\mathscr{H}^{2}$ simplifies considerably. Indeed, in this situation, $\left(\mathscr{L}^{2}\left(X^{n}\right)\right)_{n \geqslant 1}$ is a sequence of pairwise orthogonal closed subspaces of $\left(\mathscr{H}^{2},\|\cdot\|_{\mathscr{H}^{2}}\right)$. This implies that the orthogonal sum $\mathscr{H}:=\bigoplus_{n=1}^{\infty} \mathscr{L}^{2}\left(X^{n}\right)$ is closed and $\mathscr{H}$ is a stable subspace of $\mathscr{H}^{2}$. Introducing $\mathscr{C}$ as the family of all $\left(X^{n}\right)^{T}$ with stopping times $T$ such that $\left(X^{n}\right)^{T} \in \mathscr{H}^{2}$ and $n \geqslant 1$, from (4) it follows $\mathscr{C} \subseteq \mathscr{H}$. But $\mathscr{L}^{2}(\mathscr{X})$ is the smallest stable subspace of $\mathscr{H}^{2}$ containing $\mathscr{C}$ and therefore $\mathscr{L}^{2}(\mathscr{X}) \subseteq \mathscr{H}$ holds. Because of Theorem 2.1 (ii), the converse inclusion is clear and so we have proven the identity

$$
\mathscr{L}^{2}\left(\left\{X^{n}, n \geqslant 1\right\}\right)=\bigoplus_{n=1}^{\infty} \mathscr{L}^{2}\left(X^{n}\right),
$$

in case the countable family $\mathscr{X}:=\left\{X^{n}, n \geqslant 1\right\} \subseteq \mathscr{H}_{\text {loc }}^{2}$ consists of orthogonal local martingales.

Now we are ready to give the definition of the PRP. Let $\mathscr{X} \subseteq \mathscr{H}_{\mathrm{loc}}^{2}(\mathbb{F})$. We say that $\mathscr{X}$ has the PRP with respect (or relative) to $\mathbb{F}$ if

$$
\mathscr{L}^{2}(\mathscr{X})=\mathscr{H}^{2}(\mathbb{F})
$$

Let $\mathscr{X} \subseteq \mathscr{H}_{\text {loc }}^{2}$. The PRP of $\mathscr{X}$ can be characterized looking at the set of the martingales in $\mathscr{H}_{\text {loc }}^{2}$ which are orthogonal to $\mathscr{X}$. We say that $\mathscr{X} \subseteq$ $\mathscr{H}_{\text {loc }}^{2}$ satisfies condition $C_{2}$ if each martingale $Y \in \mathscr{H}^{2}$ (or, by localization, $Y \in \mathscr{H}_{\text {loc }}^{2}$ ) which is orthogonal to $\mathscr{X}$ is evanescent. For the following result cf. [13, Theorem 4.11].

Proposition 2.1. A family $\mathscr{X} \subseteq \mathscr{H}_{\text {loc }}^{2}$ possesses the PRP relative to $\mathbb{F}$ if and only if $\mathscr{X}$ satisfies condition $C_{2}$. 
3. Compensated-covariation stable families. In this section we consider a family $\mathscr{X}$ of locally square integrable local martingales and we look for sufficient conditions to ensure that the stable subspace generated by $\mathscr{X}$ in $\mathscr{H}^{2}$ equals $\mathscr{H}^{2}$ itself, i.e., that $\mathscr{X}$ possesses the PRP. The reference filtration will be the smallest filtration satisfying the usual conditions with respect to which $\mathscr{X}$ is a family of adapted processes.

We start by considering a complete probability space $(\Omega, \mathscr{F}, \mathbb{P})$ and a filtration $\mathbb{F}$ satisfying the usual conditions. Let $I$ be an arbitrary index set and $\mathscr{X} \subseteq \mathscr{H}_{\text {loc }}^{2}(\mathbb{F})$ a family of $\mathbb{F}$-local martingales indexed on $I$, i.e., $\mathscr{X}:=\left\{X^{\alpha}, \alpha \in I\right\}$. For $\alpha, \beta \in I$, we define the process

$$
X^{\alpha, \beta}:=\left[X^{\alpha}, X^{\beta}\right]-\left\langle X^{\alpha}, X^{\beta}\right\rangle
$$

and we call it the compensated-covariation process of $X^{\alpha}$ and $X^{\beta}$. The processes $\left[X^{\alpha}, X^{\beta}\right]$ and $\left\langle X^{\alpha}, X^{\beta}\right\rangle, \alpha, \beta \in I$, belong to $\mathscr{A}_{\mathrm{loc}}$, because $X^{\alpha}, X^{\beta} \in$ $\mathscr{H}_{\text {loc }}^{2}$, and the predictable process $\left\langle X^{\alpha}, X^{\beta}\right\rangle$ is the compensator of $\left[X^{\alpha}, X^{\beta}\right]$. Therefore, in general, the process $X^{\alpha, \beta}$ belongs only to $\mathscr{M}_{\mathrm{loc}}$ and not to $\mathscr{H}_{\mathrm{loc}}^{2}$. This fact justifies the following definition.

D e f i n it i o n 3.1. (i) We say that $\mathscr{X}:=\left\{X^{\alpha}, \alpha \in I\right\} \subseteq \mathscr{H}_{\text {loc }}^{2}$ is a compensated-covariation stable family if the compensated-covariation process $X^{\alpha, \beta}$ belongs to $\mathscr{X}$, for every $\alpha$ and $\beta$ in $I$.

(ii) Let $\mathscr{X}$ be a compensated-covariation stable family and let $\alpha_{1}, \ldots, \alpha_{m} \in I, m \geqslant 2$. We define the process $X^{\alpha_{1}, \ldots, \alpha_{m}}$ recursively by

$$
X^{\alpha_{1}, \ldots, \alpha_{m}}:=\left[X^{\alpha_{1}, \ldots, \alpha_{m-1}}, X^{\alpha_{m}}\right]-\left\langle X^{\alpha_{1}, \ldots, \alpha_{m-1}}, X^{\alpha_{m}}\right\rangle .
$$

The process $X^{\alpha_{1}, \ldots, \alpha_{m}}$ is defined by (9) only for $m \geqslant 2$ and this does not cause ambiguity of notation with the case $m=1$. We notice that, if $\mathscr{X}$ is a compensated-covariation stable family of local martingales, then the process $X^{\alpha_{1}, \ldots, \alpha_{m}}$ introduced in (9) is well defined and belongs to $\mathscr{X}$, for every $\alpha_{1}, \ldots, \alpha_{m} \in I, m \geqslant 2$.

Let $\alpha_{1}, \ldots, \alpha_{m} \in I, m \geqslant 2$. For notational convenience we introduce the following abbreviation:

$$
A^{\alpha_{1}, \ldots, \alpha_{m-1} \mid \alpha_{m}}:=\left\langle X^{\alpha_{1}, \ldots, \alpha_{m-1}}, X^{\alpha_{m}}\right\rangle
$$

For any family $\mathscr{X} \subseteq \mathscr{H}_{\text {loc }}^{2}$ indexed on $I$ and any collection of finitevalued stopping times $\mathscr{S}$, we define the family $\mathscr{X}^{\mathscr{S}}$ by

$$
\mathscr{X}^{\mathscr{S}}:=\left\{X^{\alpha T}, T \in \mathscr{S}, \alpha \in I\right\}
$$

It is clear that $\mathscr{X}^{\mathscr{S}} \subseteq \mathscr{H}_{\text {loc }}^{2}$. The following lemma is an easy consequence of the properties of the brackets $[\cdot, \cdot]$ and $\langle\cdot, \cdot\rangle$. Its proof is straightforward and therefore omitted. By « $\gg$ we denote the operation minimum. 
Lemma 3.1. Let $\mathscr{X}$ be a compensated-covariation stable family of $\mathscr{H}_{\mathrm{loc}}^{2}$ and $\mathscr{S}$ a family of finite-valued stopping times such that $T \wedge S \in \mathscr{S}$ for every $T$ and $S$ belonging to $\mathscr{S}$. Then the family $\mathscr{X}^{\mathscr{S}}$ introduced in (11) is a compensated-covariation stable family of local martingales in $\mathscr{H}_{\mathrm{loc}}^{2}$.

For a compensated-covariation stable family in $\mathscr{H}_{\mathrm{loc}}^{2}$ we can obtain a useful representation formula for products of its elements which is important for the further development.

Proposition 3.1. Let $\mathscr{X}:=\left\{X^{\alpha}, \alpha \in I\right\}$ be a compensated-covariation stable family of local martingales contained in $\mathscr{H}_{\mathrm{loc}}^{2}$. For every $m \in \mathbb{N}$ and for every $\alpha_{1}, \ldots, \alpha_{m} \in I$, we have

$$
\begin{aligned}
& \prod_{i=1}^{m} X^{\alpha_{i}}=\sum_{i=1}^{m} \sum_{1 \leqslant j_{1}<\cdots<j_{i} \leqslant m}\left(\prod_{\substack{k=1 \\
k \neq j_{1}, \ldots, j_{i}}}^{m} X_{-}^{\alpha_{k}}\right) \cdot X^{\alpha_{j_{1}}, \ldots, \alpha_{j_{i}}} \\
& +\sum_{p=0}^{m-2} \sum_{i=p+2}^{m} \sum_{1 \leqslant j_{1}<\cdots<j_{i} \leqslant m}\left(\prod_{\substack{k=1 \\
k \neq j_{1}, \ldots, j_{i}}}^{m} X_{-}^{\alpha_{k}} \prod_{\ell=i-p+1}^{i} \Delta X^{\alpha_{j_{\ell}}}\right) \\
& \cdots A^{\alpha_{j_{1}}, \ldots, \alpha_{j_{i-p-1}} \mid \alpha_{j_{i-p}},}
\end{aligned}
$$

where we used the notation introduced in (10).

We remark that if $\mathscr{X}$ is a compensated-covariation stable family consisting of quasi-left continuous local martingales, then formula (12) simplifies considerably. Indeed, according to [14, Theorem I.4.2], in this case we can choose a continuous version of the process $\left\langle X^{\alpha}, X^{\beta}\right\rangle, \alpha, \beta \in I$, and so all the terms appearing in the second summation on the right-hand side of (12) vanish for $p \neq 0$.

$\mathrm{P}$ r o o f. We show (12) by induction as a consequence of the formula of integration by parts (cf. [13, Theorem 2.53]). We stress that $X_{-}^{\alpha}, \alpha \in I$, is locally bounded because $X_{0}^{\alpha}=0$. Hence each summand in the first term on the right-hand side of (12) is a local martingale. From Lemma 2.1, every summand in the second term appearing on the right-hand side of (12) is a process of finite variation. Therefore the right-hand side of (12) is well defined and is a semimartingale. By convention, $\prod_{\varnothing}=1$. After these preliminary remarks, we start with the proof by induction. If $m=1$ there is nothing to prove. For the inductive step, we assume (12) for an arbitrary but fixed $m$ and we show it for $m+1$. From the formula of integration by parts, it follows

$$
\prod_{i=1}^{m+1} X^{\alpha_{i}}=\left(\prod_{i=1}^{m} X_{-}^{\alpha_{i}}\right) \cdot X^{\alpha_{m+1}}+X_{-}^{\alpha_{m+1}} \cdot \prod_{i=1}^{m} X^{\alpha_{i}}+\left[\prod_{i=1}^{m} X^{\alpha_{i}}, X^{\alpha_{m+1}}\right] .
$$

In what follows we use the notation introduced in (10). By the induction hypothesis, we can explicitly compute the second and the third terms on the 
right-hand side of (13). Indeed, for the second summand, by the induction hypothesis and the fact that $K \cdot(H \cdot X)=(H K) \cdot X$, for every $X \in \mathscr{H}_{\text {loc }}^{2}$ and locally bounded predictable $H, K$, recalling that every summand in the second summation appearing on the right-hand side of (12) is a process of finite variation (cf. Lemma 2.1 using that $X_{-}^{\alpha}$ is locally bounded, for every $\alpha \in I$ ), from (1) we get

$$
\begin{gathered}
X_{-}^{\alpha_{m+1}} \cdot \prod_{i=1}^{m} X^{\alpha_{i}}=\sum_{i=1}^{m} \sum_{\substack{1 \leqslant j_{1}<\cdots<j_{i} \leqslant m \\
+}}\left(X_{-}^{\alpha_{m+1}} \prod_{\substack{k=1 \\
k \neq j_{1}, \ldots, j_{i}}}^{m} X_{-}^{\alpha_{k}}\right) \cdot X^{\alpha_{j_{1}}, \ldots, \alpha_{j_{i}}} \\
\sum_{p=0}^{m-2} \sum_{i=p+2}^{m} \sum_{1 \leqslant j_{1}<\cdots<j_{i} \leqslant m}\left(X_{-}^{\alpha_{m+1}} \prod_{\substack{k=1 \\
k \neq j_{1}, \ldots, j_{i} \\
\alpha_{j_{1}}, \ldots, \alpha_{j_{i-p-1}} \mid \alpha_{j_{i-p}}}}^{m} X_{-}^{\alpha_{k}} \prod_{\ell=i-p+1}^{i} \Delta X^{\alpha_{j_{\ell}}}\right) \\
\cdots A^{\alpha_{1}}
\end{gathered}
$$

Now we discuss the third summand appearing on the right-hand side of formula (13). For any $1 \leqslant j_{1}<\cdots<j_{i} \leqslant m$, where $1 \leqslant i \leqslant m$ is given, we have that the integrals $\left(\prod_{k \neq j_{1}, \ldots, j_{i}}^{m} X_{-}^{\alpha_{k}}\right)\left(\prod_{\ell=i-p+1}^{i} \Delta X^{\alpha_{j_{\ell}}}\right) \cdot A^{\alpha_{j_{1}}, \ldots, \alpha_{j_{i-p-1}} \mid \alpha_{j_{i-p}}}$ belong to $\mathscr{V}$. By the induction hypothesis and (5) we have

$$
\begin{aligned}
& {\left[\prod_{i=1}^{m} X^{\alpha_{i}}, X^{\alpha_{m+1}}\right]=\sum_{i=1}^{m} \sum_{1 \leqslant j_{1}<\cdots<j_{i} \leqslant m}\left(\prod_{\substack{k=1 \\
k \neq j_{1}, \ldots, j_{i}}}^{m} X_{-}^{\alpha_{k}}\right) \cdot\left[X^{\alpha_{j_{1}}, \ldots, \alpha_{j_{i}}}, X^{\alpha_{m+1}}\right]} \\
& +\sum_{p=0}^{m-2} \sum_{i=p+2}^{m} \sum_{1 \leqslant j_{1}<\cdots<j_{i} \leqslant m}\left(\prod_{\substack{k=1 \\
k \neq j_{1}, \ldots, j_{i}}}^{m} X_{-}^{\alpha_{k}} \prod_{\ell=i-p+1}^{i} \Delta X^{\alpha_{j_{\ell}}}\right) \\
& \cdots\left[A^{\alpha_{j_{1}}, \ldots, \alpha_{j_{i-p-1}} \mid \alpha_{j_{i-p}}}, X^{\alpha_{m+1}}\right] .
\end{aligned}
$$

From (3) and (1), we can conclude that

$$
\begin{gathered}
{\left[\prod_{i=1}^{m} X^{\alpha_{i}}, X^{\alpha_{m+1}}\right]=\sum_{i=1}^{m} \sum_{1 \leqslant j_{1}<\cdots<j_{i} \leqslant m}\left(\prod_{\substack{k=1 \\
k \neq j_{1}, \ldots, j_{i}}}^{m} X_{-}^{\alpha_{k}}\right) \cdot\left[X^{\alpha_{j_{1}}, \ldots, \alpha_{j_{i}}}, X^{\alpha_{m+1}}\right]} \\
+\sum_{p=0}^{m-2} \sum_{i=p+2}^{m} \sum_{1 \leqslant j_{1}<\cdots<j_{i} \leqslant m}\left(\prod_{\substack{k=1 \\
k \neq j_{1}, \ldots, j_{i}}}^{m} X_{-}^{\alpha_{k}} \Delta X^{\alpha_{m+1}} \prod_{\ell=i-p+1}^{i} \Delta X^{\alpha_{j_{\ell}}}\right) \\
\cdots A^{\alpha_{j_{1}}, \ldots, \alpha_{j_{i-p-1}} \mid \alpha_{j_{i-p}} .}
\end{gathered}
$$

Using the linearity of the Riemann-Stieltjes integral and (9) in the first term on the right-hand side of the previous formula, it follows:

$$
\left[\prod_{i=1}^{m} X^{\alpha_{i}}, X^{\alpha_{m+1}}\right]=\sum_{i=1}^{m} \sum_{1 \leqslant j_{1}<\cdots<j_{i} \leqslant m}\left(\prod_{\substack{k=1 \\ k \neq j_{1}, \ldots, j_{i}}}^{m} X_{-}^{\alpha_{k}}\right) \cdot X^{\alpha_{j_{1}}, \ldots, \alpha_{j_{i}}, \alpha_{m+1}}
$$




$$
\begin{aligned}
& +\sum_{i=1}^{m} \sum_{1 \leqslant j_{1}<\cdots<j_{i} \leqslant m}\left(\prod_{\substack{k=1 \\
k \neq j_{1}, \ldots, j_{i}}}^{m} X_{-}^{\alpha_{k}}\right) \cdot A^{\alpha_{j_{1}}, \ldots, \alpha_{j_{i}} \mid \alpha_{m+1}} \\
& +\sum_{p=0}^{m-2} \sum_{i=p+2}^{m} \sum_{1 \leqslant j_{1}<\cdots<j_{i} \leqslant m}\left(\prod_{\substack{k=1 \\
k \neq j_{1}, \ldots, j_{i}}}^{m} X_{-}^{\alpha_{k}} \Delta X^{\alpha_{m+1}} \prod_{\ell=i-p+1}^{i} \Delta X^{\alpha_{j_{\ell}}}\right) \\
& \text {. } A^{\alpha_{j_{1}}, \ldots, \alpha_{j_{i-p-1}} \mid \alpha_{j_{i-p}}} \text {. }
\end{aligned}
$$

Inserting (14) and (15) in (13) we get

$$
\begin{aligned}
& \prod_{i=1}^{m+1} X^{\alpha_{i}}=\left(\prod_{i=1}^{m} X_{-}^{\alpha_{i}}\right) \cdot X^{\alpha_{m+1}} \\
& +\sum_{i=1}^{m} \sum_{1 \leqslant j_{1}<\cdots<j_{i} \leqslant m}\left(X_{-}^{\alpha_{m+1}} \prod_{\substack{k=1 \\
k \neq j_{1}, \ldots, j_{i}}}^{m} X_{-}^{\alpha_{k}}\right) \cdot X^{\alpha_{j_{1}}, \ldots, \alpha_{j_{i}}} \\
& +\sum_{i=1}^{m} \sum_{1 \leqslant j_{1}<\cdots<j_{i} \leqslant m}\left(\prod_{\substack{k=1 \\
k \neq j_{1}, \ldots, j_{i}}}^{m} X_{-}^{\alpha_{k}}\right) \cdot X^{\alpha_{j_{1}}, \ldots, \alpha_{j_{i}}, \alpha_{m+1}} \\
& +\sum_{p=0}^{m-2} \sum_{i=p+2}^{m} \sum_{1 \leqslant j_{1}<\cdots<j_{i} \leqslant m}\left(X_{-}^{\alpha_{m+1}} \prod_{\substack{k=1 \\
k \neq j_{1}, \ldots, j_{i}}}^{m} X_{-}^{\alpha_{k}} \prod_{\ell=i-p+1}^{i} \Delta X^{\alpha_{j_{\ell}}}\right) \\
& \cdots A^{\alpha_{j_{1}}, \ldots, \alpha_{j_{i-p-1}} \mid \alpha_{j_{i-p}}} \\
& +\sum_{i=1}^{m} \sum_{1 \leqslant j_{1}<\cdots<j_{i} \leqslant m}\left(\prod_{\substack{k=1 \\
k \neq j_{1}, \ldots, j_{i}}}^{m+1} X_{-}^{\alpha_{k}}\right) \cdot A^{\alpha_{j_{1}}, \ldots, \alpha_{j_{i}} \mid \alpha_{m+1}} \\
& +\sum_{p=0}^{m-2} \sum_{i=p+2}^{m} \sum_{1 \leqslant j_{1}<\cdots<j_{i} \leqslant m}\left(\prod_{\substack{k=1 \\
k \neq j_{1}, \ldots, j_{i}}}^{m} X_{-}^{\alpha_{k}} \Delta X^{\alpha_{m+1}} \prod_{\ell=i-p+1}^{i} \Delta X^{\alpha_{j_{\ell}}}\right) \\
& \text {... } A^{\alpha_{j_{1}}, \ldots, \alpha_{j_{i-p-1}} \mid \alpha_{j_{i-p}}} \text {. }
\end{aligned}
$$

Let us now discuss (16), (17), and (18). We can rewrite (18) as follows:

$$
(18)=\sum_{i=2}^{m+1} \sum_{\substack{1 \leqslant j_{1}<\ldots<j_{i} \leqslant m+1 \\ j_{i}=m+1}}\left(\prod_{\substack{k=1 \\ k \neq j_{1}, \ldots, j_{i}}}^{m+1} X_{-}^{\alpha_{k}}\right) \cdot X^{\alpha_{j_{1}}, \ldots, \alpha_{j_{i}}}
$$

while (16) is the term for $i=1$. Putting together (16) and (18), we obtain

$$
(16)+(18)=\sum_{i=1}^{m+1} \sum_{\substack{1 \leqslant j_{1}<\ldots<j_{i} \leqslant m+1 \\ j_{i}=m+1}}\left(\prod_{\substack{k=1 \\ k \neq j_{1}, \ldots, j_{i}}}^{m+1} X_{-}^{\alpha_{k}}\right) \cdot X^{\alpha_{j_{1}}, \ldots, \alpha_{j_{i}}}
$$

Furthermore, (17) can be written as

$$
(17)=\sum_{i=1}^{m+1} \sum_{\substack{1 \leqslant j_{1}<\ldots<j_{i} \leqslant m+1 \\ j_{i} \neq m+1}}\left(\prod_{\substack{k=1 \\ k \neq j_{1}, \ldots, j_{i}}}^{m+1} X_{-}^{\alpha_{k}}\right) \cdot X^{\alpha_{j_{1}}, \ldots, \alpha_{j_{i}}}
$$


and this yields

$$
(16)+(17)+(18)=\sum_{i=1}^{m+1} \sum_{1 \leqslant j_{1}<\cdots<j_{i} \leqslant m+1}\left(\prod_{\substack{k=1 \\ k \neq j_{1}, \ldots, j_{i}}}^{m+1} X_{-}^{\alpha_{k}}\right) \cdot X^{\alpha_{j_{1}}, \ldots, \alpha_{j_{i}}}
$$

which is the first summand on the right-hand side of (12) for $m+1$. Now we come to the discussion of (19), (20), and (21). For (20) we have

$$
(20)=\sum_{i=2}^{m+1} \sum_{\substack{1 \leqslant j_{1}<\ldots<j_{i} \leqslant m+1 \\ j_{i}=m+1}}\left(\prod_{\substack{k=1 \\ k \neq j_{1}, \ldots, j_{i}}}^{m+1} X_{-}^{\alpha_{k}}\right) \cdot A^{\alpha_{j_{1}}, \ldots, \alpha_{j_{i-1}} \mid \alpha_{j_{i}}}
$$

Setting $p^{\prime}:=p+1$ and denoting it again by $p$ yields

$$
\begin{gathered}
(21)=\sum_{p=1}^{(m+1)-2} \sum_{\substack { i=p+2 \\
\begin{subarray}{c}{1 \leqslant j_{1}<\cdots<j_{i} \leqslant m+1 \\
j_{i}=m+1{ i = p + 2 \\
\begin{subarray} { c } { 1 \leqslant j _ { 1 } < \cdots < j _ { i } \leqslant m + 1 \\
j _ { i } = m + 1 } }\end{subarray}}\left(\prod_{\substack{k=1 \\
k \neq j_{1}, \ldots, j_{i}}}^{m+1} X_{-}^{\alpha_{k}} \prod_{\ell=i-p+1}^{i} \Delta X^{\alpha_{j_{\ell}}}\right) \\
\cdots A^{\alpha_{j_{1}}, \ldots, \alpha_{j_{i-p-1}} \mid \alpha_{j_{i-p}}} .
\end{gathered}
$$

Putting together (20) and (21) we obtain

$$
\begin{gathered}
(20)+(21)=\sum_{p=0}^{m-1} \sum_{\substack { i=p+2 \\
\begin{subarray}{c}{1 \leqslant j_{1}<\cdots<j_{i} \leqslant m+1 \\
j_{i}=m+1{ i = p + 2 \\
\begin{subarray} { c } { 1 \leqslant j _ { 1 } < \cdots < j _ { i } \leqslant m + 1 \\
j _ { i } = m + 1 } }\end{subarray}}\left(\prod_{\substack{k=1 \\
k \neq j_{1}, \ldots, j_{i} \\
\cdots}}^{m+1} X_{-}^{\alpha_{k}} \prod_{\ell=i-p+1}^{i} \Delta X^{\alpha_{j_{\ell}}}\right) \\
\cdots, A^{\alpha_{j_{1}}, \ldots, \alpha_{j_{i-p-1}} \mid \alpha_{j_{i-p}}} .
\end{gathered}
$$

Finally, (19) can be written as

$$
\begin{gathered}
(19)=\sum_{p=0}^{(m+1)-2} \sum_{\substack { i=p+2 \\
\begin{subarray}{c}{1 \leqslant j_{1}<\cdots<j_{i} \leqslant m+1 \\
j_{i} \neq m+1{ i = p + 2 \\
\begin{subarray} { c } { 1 \leqslant j _ { 1 } < \cdots < j _ { i } \leqslant m + 1 \\
j _ { i } \neq m + 1 } }\end{subarray}}\left(\prod_{\substack{k=1 \\
k \neq j_{1}, \ldots, j_{i}}}^{m+1} X_{-}^{\alpha_{k}} \prod_{\ell=i-p+1}^{i} \Delta X^{\alpha_{j_{\ell}}}\right) \\
\cdots A^{\alpha_{j_{1}}, \ldots, \alpha_{j_{i-p-1}} \mid \alpha_{j_{i-p}}} .
\end{gathered}
$$

We notice that in the previous formula we get that $\left\{j_{1}, \ldots, j_{i}\right\}$ is equal to the empty set when $p=(m+1)-2$. Indeed, this case for $p$ yields $i=m+1$ but we have, from $j_{i} \neq m+1$, that the case $\left\{j_{1}, \ldots, j_{i}\right\}$ with $i=m+1$ is impossible. As a result, we get that $(19)+(20)+(21)$ is equal to

$$
\sum_{p=0}^{m-1} \sum_{i=p+2}^{m+1} \sum_{1 \leqslant j_{1}<\cdots<j_{i} \leqslant m+1}\left(\prod_{\substack{k=1 \\ k \neq j_{1}, \ldots, j_{i}}}^{m+1} X_{-}^{\alpha_{k}} \prod_{\ell=i-p+1}^{i} \Delta X^{\alpha_{j_{\ell}}}\right) \cdot A^{\alpha_{j_{1}}, \ldots, \alpha_{j_{i-p-1}} \mid \alpha_{j_{i-p}}} .
$$

This is the second summand on the right-hand side of (12) for $m+1$ and the proof is finished. Proposition 3.1 is proved.

Let $\mathscr{X}:=\left\{X^{\alpha}, \alpha \in I\right\}$ be a family of local martingales in $\mathscr{H}_{\mathrm{loc}}^{2}$ (not necessarily compensated-covariation stable). By $\mathscr{F}_{\infty}^{\mathscr{X}}$ we denote the 
$\mathbb{P}$-completion of the $\sigma$-algebra $\sigma\left(X_{t}^{\alpha}, t \geqslant 0, \alpha \in I\right)$ in itself, i.e., $\mathscr{F}_{\infty}^{\mathscr{X}}:=$ $\sigma\left(X_{t}^{\alpha}, t \geqslant 0, \alpha \in I\right) \vee \mathscr{N}(\mathbb{P})$, where $\mathscr{N}(\mathbb{P})$ indicates the null-sets of $\mathbb{P}$ with respect to $\sigma\left(X_{t}^{\alpha}, t \geqslant 0, \alpha \in I\right)$. On the probability space $\left(\Omega, \mathscr{F}_{\infty} \mathscr{X}, \mathbb{P}\right)$, we denote by $\mathbb{F}^{\mathscr{X}}$ the smallest filtration satisfying the usual conditions (completion in $\mathscr{F}_{\infty}^{\mathscr{X}}$ ) such that $\mathscr{X}$ is an adapted family. From now on, we restrict our attention to the probability space $\left(\Omega, \mathscr{F}_{\infty}^{\mathscr{X}}, \mathbb{P}\right)$ and to the filtration $\mathbb{F}^{\mathscr{X}}$, i.e., $\mathscr{X}$ is a subfamily of $\mathscr{H}_{\text {loc }}^{2}\left(\mathbb{F}^{\mathscr{X}}\right)$ on the probability space $\left(\Omega, \mathscr{F}_{\infty}^{\mathscr{X}}, \mathbb{P}\right)$.

In the remaining part of this section, we look for sufficient conditions to ensure that $\mathscr{X}$ possesses the PRP with respect to $\mathbb{F}^{\mathscr{X}}$. The first assumption which we need concerns the existence of moments for the elements of $\mathscr{X}$. We assume that $\mathscr{X} \subseteq \mathscr{H}_{\text {loc }}^{2}$ is such that $X_{t}^{\alpha}$ admits a finite moment of every order, i.e.,

$$
\mathbb{E}\left[\left|X_{t}^{\alpha}\right|^{n}\right]<+\infty, \quad t \geqslant 0, \quad \alpha \in I, \quad n \in \mathbb{N} .
$$

If $\mathscr{X}$ is a family of true martingales contained in $\mathscr{H}^{2}$ we can replace $(22)$ by the following assumption:

$$
\mathbb{E}\left[\left|X_{\infty}^{\alpha}\right|^{n}\right]<+\infty, \quad \alpha \in I, \quad n \in \mathbb{N}
$$

Note that (23) is stronger than (22). We now recall the well-known estimation of the geometric mean by the arithmetic mean, which will be used in the sequel. For every $a_{1}, \ldots, a_{m} \geqslant 0, m \in \mathbb{N}$, it follows:

$$
\left(\prod_{i=1}^{m} a_{i}\right)^{1 / m} \leqslant \frac{1}{m} \sum_{i=1}^{m} a_{i}
$$

We define the family $\mathscr{K}$ by

$$
\mathscr{K}:=\left\{\prod_{i=1}^{m} X_{t_{i}}^{\alpha_{i}}: \alpha_{i} \in I, t_{i} \in \mathbb{R}, i=1, \ldots, m, m \in \mathbb{N}\right\}
$$

which is the family of monomials generated by products of elements of $\mathscr{X}$ at different times. If $\mathscr{X} \subseteq \mathscr{H}_{\mathrm{loc}}^{2}$ satisfies (22), then, as a consequence of (24), we get $\mathscr{K} \subseteq L^{2}\left(\Omega, \mathscr{F}_{\infty}^{\mathscr{X}}, \mathbb{P}\right)$. Obviously, we have $\sigma(\mathscr{K}) \vee \mathscr{N}(\mathbb{P})=\mathscr{F} \mathscr{\infty}$. We make the following assumption.

Assumption 3.1. The family $\mathscr{K} \subseteq L^{2}\left(\Omega, \mathscr{F}_{\infty}^{\mathscr{X}}, \mathbb{P}\right)$ defined in (25) is total (i.e., its linear hull is dense) in $L^{2}\left(\Omega, \mathscr{F}_{\infty}^{\mathscr{X}}, \mathbb{P}\right)$.

It is possible to give sufficient conditions on the family $\mathscr{X}$ to be such that $\mathscr{K}$ is total in the Hilbert space $L^{2}\left(\Omega, \mathscr{F}_{\infty}^{\mathscr{X}}, \mathbb{P}\right)$ as the following wellknown theorem states.

Theorem 3.1. If for every $\alpha \in I$ the martingale $X^{\alpha}$ is such that for every $t \geqslant 0$ there exists a constant $c_{\alpha}(t)>0$ such that $\mathbb{E}\left[\exp \left(c_{\alpha}(t)\left|X_{t}^{\alpha}\right|\right)\right]<$ $+\infty, t \geqslant 0$, then $\mathscr{K} \subseteq L^{2}\left(\Omega, \mathscr{F}_{\infty}^{\mathscr{X}}, \mathbb{P}\right)$ and it satisfies Assumption 3.1. 
In the appendix (cf. Theorem A.1) we provide a simple proof of Theorem 3.1.

Before coming to the discussion of the PRP, we need some technical preparation. We begin with the following lemma.

Lemma 3.2. Let $A \in \mathscr{A}$ be a deterministic process; $\mathscr{X}:=\left\{X^{\alpha}, \alpha \in I\right\}$ a family of martingales contained in $\mathscr{H}^{2}$ satisfying $(23)$ and $m, p, q \in \mathbb{N}$. For $\alpha_{i}, \beta_{j} \in I, i=1, \ldots, m, j=1, \ldots, q$, we define the processes $H$ and $K$ by

$$
H:=\prod_{i=1}^{m}\left|X_{-}^{\alpha_{i}}\right|^{p}, \quad K:=\left(\prod_{j=1}^{q} \Delta X^{\beta_{j}} \prod_{i=1}^{m} X_{-}^{\alpha_{i}}\right) .
$$

Then the processes $H \cdot A$ and $K \cdot A$ belong to $\mathscr{A}$.

$\mathrm{P}$ r o o f. Note that, because of Lemma 2.1, recalling that $X_{-}^{\alpha}$ is locally bounded for every $\alpha \in I$, both the processes $H \cdot A$ and $K \cdot A$ are well defined and belong to $\mathscr{V}$. Furthermore, because $A \in \mathscr{A}$ is deterministic, the terminal variable $A_{\infty}$ is finite valued. The proof that $K \cdot A$ is of integrable variation is similar to the one that $H \cdot A$ is of integrable variation. We verify the statement only for $H \cdot A$. In a first step we assume that $A \in \mathscr{A}^{+}$. As a consequence of Fubini's theorem we have $\mathbb{E}\left[H \cdot A_{\infty}\right]=\mathbb{E}[H] \cdot A_{\infty}$, where by $\mathbb{E}[H]$ we denote the function $t \mapsto \mathbb{E}\left[H_{t}\right], t \geqslant 0$. Hence, by $(24)$,

$$
\mathbb{E}\left[H \cdot A_{\infty}\right]=\mathbb{E}[H] \cdot A_{\infty} \leqslant \sum_{i=1}^{m} \mathbb{E}\left[\left|X_{\infty}^{\alpha_{i}}\right|^{p m}\right] A_{\infty}<\infty,
$$

where in the last estimation we used that $\mathscr{X} \subseteq \mathscr{H}^{2}$ satisfies (23). If $A \in \mathscr{A}$ we have that $H \cdot A \in \mathscr{V}$ and $\operatorname{Var}(H \cdot A)_{\infty}=H \cdot \operatorname{Var}(A)_{\infty}$. Thus, from the previous step we get $H \cdot A \in \mathscr{A}$. Lemma 3.2 is proved.

From Lemma 3.2, we can immediately deduce that, if $\mathscr{X} \subseteq \mathscr{H}^{2}$ satisfies (23) and $X^{\alpha} \in \mathscr{X}$ is such that $\left\langle X^{\alpha}, X^{\alpha}\right\rangle$ is deterministic, then the predictable process $\prod_{i=1}^{m} X_{-}^{\alpha_{i}}$ belongs to $\mathrm{L}^{2}\left(X^{\alpha}\right)$, for every $\alpha, \alpha_{1}, \ldots, \alpha_{m} \in I$.

Lemma 3.3. Let $\mathscr{X} \subseteq \mathscr{H}^{2}$ be a family of martingales which satisfies $(23) ; q, r \in \mathbb{N}$ fixed and $\xi \in L^{2}(\mathbb{P})$ such that, for some $\alpha_{1}, \ldots, \alpha_{q+r} \in I$, $\xi$ is orthogonal in $L^{2}(\mathbb{P})$ to $\prod_{k=1}^{q+r} X_{t_{k}}^{\alpha_{k}}$ for every $t_{1}, \ldots, t_{q+r} \in \mathbb{R}_{+}$. Then we also have that $\xi$ is orthogonal to $\prod_{k=1}^{q} X_{t-}^{\alpha_{k}} \prod_{j=q+1}^{q+r} X_{t}^{\alpha_{j}}$ for every $t \in \mathbb{R}_{+}$.

P r o o f. Let $\left(t_{n}\right)_{n \geqslant 1} \subseteq \mathbb{R}_{+}$be such that $t_{n} \uparrow t$. Setting $a_{n}:=\prod_{k=1}^{q} X_{t_{n}}^{\alpha_{k}}$, then $a_{n}$ converges to $\prod_{k=1}^{q} X_{t-}^{\alpha_{k}}$ a.s. for $n \rightarrow+\infty$. It is clear that, for every fixed $n \geqslant 1, \xi$ is orthogonal to $b_{n}:=a_{n} \prod_{j=q+1}^{q+r} X_{t}^{\alpha_{j}}$. We now show that the sequence $\left(c_{n}\right)_{n \geqslant 1}$, where $c_{n}:=b_{n} \xi, n \geqslant 1$, is dominated in $L^{1}(\mathbb{P})$. From $(24)$ we have $\left|c_{n}\right| \leqslant \xi^{2}+b_{n}^{2}$. By assumption, $\xi \in L^{2}(\mathbb{P})$ and so it remains to show that $\left(b_{n}^{2}\right)_{n \geqslant 1}$ is dominated in $L^{1}(\mathbb{P})$. From the definition of $b_{n}$ and $(24)$, we obtain

$$
b_{n}^{2} \leqslant \sum_{k=1}^{q} \sup _{0 \leqslant s \leqslant t}\left\{\left(X_{s}^{\alpha_{k}}\right)^{4 q}\right\}+\sum_{j=q+1}^{q+r}\left(X_{t}^{\alpha_{j}}\right)^{4 r}, \quad n \geqslant 1,
$$


and, by Doob's inequality and (23), the right-hand side in the previous estimation is integrable. Hence $\left(c_{n}\right)_{n \geqslant 1}$ is dominated in $L^{1}(\mathbb{P})$ by a quantity which does not depend on $n$. From Lebesgue's dominated convergence theorem we get $0=\lim _{n \rightarrow \infty} \mathbb{E}\left[c_{n}\right]=\mathbb{E}\left[\lim _{n \rightarrow \infty} c_{n}\right]$ and this concludes the proof. Lemma 3.3 is proved.

Now we come to the main result of this paper, concerning the PRP of compensated-covariation stable families of martingales in $\mathscr{H}_{\text {loc }}^{2}$.

Theorem 3.2. Let $\mathscr{X}=\left\{X^{\alpha}, \alpha \in I\right\}$ be a compensated-covariation stable family of true martingales in $\mathscr{H}_{\mathrm{loc}}^{2}$ on the probability space $\left(\Omega, \mathscr{F}_{\infty}, \mathbb{P}\right)$ and with respect to the filtration $\mathbb{F}^{\mathscr{X}}$. If $\mathscr{X}$ satisfies (22), Assumption 3.1 and furthermore the process $\left\langle X^{\alpha}, X^{\beta}\right\rangle$ is deterministic, for every $\alpha, \beta \in I$, then the family $\mathscr{X}$ has the PRP with respect to $\mathbb{F}^{\mathscr{X}}$.

P r o o f. Let $\mathscr{S}:=\mathbb{R}_{+}$be the family of deterministic stopping times and $\mathscr{X}^{\mathscr{S}}$ the family obtained from $\mathscr{X}$ as in (11). Obviously $\mathscr{X}^{\mathscr{S}}$ is stable under stopping with respect to deterministic stopping times and it satisfies (23) because $\mathscr{X}$ satisfies (22). Hence, because $\mathscr{X} \subseteq \mathscr{H}_{\text {loc }}^{2}$ is a family of true martingales, we have that $\mathscr{X}^{\mathscr{S}}$ is in fact contained in $\mathscr{H}^{2}$. Thanks to Lemma $3.1, \mathscr{X}^{\mathscr{S}}$ is again a compensated-covariation stable family. Moreover, $\mathscr{L}^{2}\left(\mathscr{X}^{\mathscr{S}}\right)$ is equal to $\mathscr{L}^{2}(\mathscr{X})$ : First we verify that the inclusion $\mathscr{L}^{2}(\mathscr{X}) \subseteq \mathscr{L}^{2}\left(\mathscr{X}^{\mathscr{S}}\right)$ holds. As an immediate consequence of (4), $1_{[0, k]} H \cdot X^{\alpha} \in \mathscr{L}^{2}\left(\mathscr{X}^{\mathscr{S}}\right)$ for every $k \geqslant 1$, and $H \in \mathrm{L}\left(X^{\alpha}\right)$ and from [14, Theorem I.4.40 (iii')], the sequence of processes $1_{[0, k]} H \cdot X^{\alpha}$ converges in $\left(\mathscr{H}^{2},\|\cdot\|_{\mathscr{H}^{2}}\right)$ to $H \cdot X^{\alpha}$ as $k \rightarrow+\infty, H \in \mathrm{L}\left(X^{\alpha}\right), \alpha \in I$, meaning that $H \cdot X^{\alpha} \in \mathscr{L}^{2}\left(\mathscr{X}^{\mathscr{S}}\right)$, because $\mathscr{L}^{2}\left(\mathscr{X}^{\mathscr{S}}\right)$ is a stable subspace of $\mathscr{H}^{2}$. The inclusion $\mathscr{L}^{2}\left(\mathscr{X}^{\mathscr{S}}\right) \subseteq \mathscr{L}^{2}(\mathscr{X})$ is clear. This means that to show that $\mathscr{X}$ possesses the PRP it is enough to verify that $\mathscr{X}^{\mathscr{S}}$ possesses the PRP. Summarizing, $\mathscr{X}^{\mathscr{S}}$ is a compensated-covariation stable family contained in $\mathscr{H}^{2}$ satisfying (23) and the point brackets of elements of $\mathscr{X}^{\mathscr{S}}$ are deterministic. Moreover, $\mathscr{X}^{\mathscr{S}}$ is stable under stopping with respect to deterministic stopping times and generates the same stable subspace as $\mathscr{X}$. Therefore, without loss of generality, we can assume that $\mathscr{X}$ is stable under stopping with respect to deterministic stopping times, is contained in $\mathscr{H}^{2}$ and satisfies (23): If this would not be the case, we can consider the family $\mathscr{X}^{\mathscr{S}}$ in place of $\mathscr{X}$. These further assumptions on the family $\mathscr{X}$ will considerably simplify the notation in the sequel. Our aim is to show that the compensated-covariation stable family $\mathscr{X}$ fulfilling the assumptions of the statement of the theorem and the further assumptions explained before, satisfies condition $C_{2}$ (cf. the paragraph after (7)). In other words, we show that every martingale $Y$ belonging to $\mathscr{H}^{2}$ which is orthogonal to $\mathscr{X}$ is evanescent. To prove this claim we verify that if $Y \in \mathscr{H}^{2}$ is orthogonal to $\mathscr{X}$, then its terminal variable $Y_{\infty}$ is orthogonal in $L^{2}\left(\Omega, \mathscr{F}_{\infty}^{\mathscr{X}}, \mathbb{P}\right)$ to the system $\mathscr{K}$ defined in $(25)$ which is total in $L^{2}\left(\Omega, \mathscr{F}_{\infty}^{\mathscr{X}}, \mathbb{P}\right)$ by assumption. We proceed by induction on $m \in \mathbb{N}$, 
where $m$ is the order of the monomials in $\mathscr{K}$ (cf. (25)). Let $Y \in \mathscr{H}^{2}\left(\mathbb{F}^{\mathscr{X}}\right)$ be such that $Y \perp \mathscr{X}$. If $m=1$, because of the orthogonality, we get $Y X^{\alpha} \in \mathscr{M}$ and so $\mathbb{E}\left[Y_{\infty} X_{\infty}^{\alpha}\right]=0$. Now we assume that we have

$$
\mathbb{E}\left[Y_{\infty} \prod_{i=1}^{n} X_{\infty}^{\alpha_{i}}\right]=0, \quad \alpha_{1}, \ldots, \alpha_{n} \in I, \quad n \leqslant m
$$

From $(27)$ and the property that $\mathscr{X}$ is stable under stopping with respect to deterministic stopping times, it easily follows that

$$
\mathbb{E}\left[Y_{\infty} \prod_{i=1}^{n} X_{t_{i}}^{\alpha_{i}}\right]=0, \quad t_{1}, \ldots, t_{n} \in \mathbb{R}_{+}, \quad \alpha_{1}, \ldots, \alpha_{n} \in I, \quad n \leqslant m
$$

Now we prove $(27)$ for $m+1$. As $Y_{\infty} \in L^{2}\left(\Omega, \mathscr{F}_{\infty}^{\mathscr{X}}, \mathbb{P}\right)$, the product $Y_{\infty} \prod_{i=1}^{m+1} X_{\infty}^{\alpha_{i}}$ is integrable and from Proposition 3.1, using the notation introduced in (10), we have

$$
\begin{aligned}
& \mathbb{E}\left[Y_{\infty} \prod_{i=1}^{m+1} X_{\infty}^{\alpha_{i}}\right]=\sum_{i=1}^{m+1} \sum_{1 \leqslant j_{1}<\cdots<j_{i} \leqslant m+1} \mathbb{E}\left[Y_{\infty}\left(\prod_{\substack{k=1 \\
k \neq j_{1}, \ldots, j_{i}}}^{m+1} X_{-}^{\alpha_{k}}\right) \cdot X_{\infty}^{\alpha_{j_{1}}, \ldots, \alpha_{j_{i}}}\right] \\
& +\sum_{p=0}^{m-1} \sum_{i=p+2}^{m+1} \sum_{1 \leqslant j_{1}<\cdots<j_{i} \leqslant m+1} \mathbb{E}\left[Y_{\infty}\left(\prod_{\substack{k=1 \\
k \neq j_{1}, \ldots, j_{i}}}^{m+1} X_{-}^{\alpha_{k}} \prod_{\ell=i-p+1}^{i} \Delta X^{\alpha_{j_{\ell}}}\right)\right.
\end{aligned}
$$

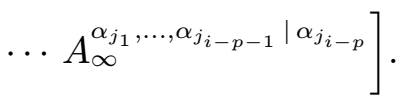

For the first term on the right-hand side of (29) we have

$$
\sum_{i=1}^{m+1} \sum_{1 \leqslant j_{1}<\cdots<j_{i} \leqslant m+1} \mathbb{E}\left[Y_{\infty}\left(\prod_{\substack{k=1 \\ k \neq j_{1}, \ldots, j_{i}}}^{m+1} X_{-}^{\alpha_{k}}\right) \cdot X_{\infty}^{\alpha_{j_{1}}, \ldots, \alpha_{j_{i}}}\right]=0
$$

Indeed, from Lemma 3.2 , it follows that $\prod_{k \neq j_{1}, \ldots, j_{i}}^{m+1} X_{-}^{\alpha_{k}}$ belongs to $\mathrm{L}^{2}\left(X^{\alpha_{j_{1}}, \ldots, \alpha_{j_{i}}}\right)$, for every fixed $1 \leqslant j_{1}<\cdots<j_{i} \leqslant m$. Hence Theorem 2.1 (i) yields that every stochastic integral with respect to $X^{\alpha_{j_{1}}, \ldots, \alpha_{j_{i}}}$ appearing in (30) belongs to the space $\mathscr{L}^{2}\left(X^{\alpha_{j_{1}}, \ldots, \alpha_{j_{i}}}\right)$. Each element $X^{\alpha_{j_{1}}, \ldots, \alpha_{j_{i}}}$ belongs again to $\mathscr{X}$, because $\mathscr{X}$ is a compensated-covariation stable family. From the discussion on page 104, after Theorem 2.1, we know that if $N \in \mathscr{H}^{2}$ is orthogonal to $X^{\alpha_{j_{1}}, \ldots, \alpha_{j_{i}}}$, then $N$ is also orthogonal to $\mathscr{L}^{2}\left(X^{\alpha_{j_{1}}, \ldots, \alpha_{j_{i}}}\right)$. This yields $N X \in \mathscr{M}$, for every $X$ belonging to $\mathscr{L}^{2}\left(X^{\alpha_{j_{1}}, \ldots, \alpha_{j_{i}}}\right)$. Therefore, for every $X \in \mathscr{L}^{2}\left(X^{\alpha_{j_{1}}, \ldots, \alpha_{j_{i}}}\right)$, we have $\mathbb{E}\left[Y_{\infty} X_{\infty}\right]=0$ and this, together with Theorem 2.1 (i), proves (30). Now we show that the second term on the righthand side of (29) vanishes. The processes $X^{\alpha_{j_{i-p}}}$ and $X^{\alpha_{j_{1}}, \ldots, \alpha_{j_{i-p-1}}}$ belong to $\mathscr{X}$ for every $i$ and $p$, because $\mathscr{X}$ is compensated-covariation stable. By assumption, the processes $A^{\alpha_{j_{1}}, \ldots, \alpha_{j_{i-p-1}} \mid \alpha_{j_{i-p}}}$ are deterministic. Lemma 3.2 
implies that $\left(\prod_{k \neq j_{1}, \ldots, j_{i}}^{m+1} X_{-}^{\alpha_{k}}\right)\left(\prod_{\ell=i-p+1}^{i} \Delta X^{\alpha_{j_{\ell}}}\right) \cdot A^{\alpha_{j_{1}}, \ldots, \alpha_{j_{i-p-1}} \mid \alpha_{j_{i-p}}}$ are processes of integrable variation. Thus we can apply Fubini's theorem and get

$$
\begin{gathered}
\sum_{p=0}^{m-1} \sum_{i=p+2}^{m+1} \sum_{1 \leqslant j_{1}<\cdots<j_{i} \leqslant m+1} \mathbb{E}\left[Y_{\infty}\left(\prod_{\substack{k=1 \\
k \neq j_{1}, \ldots, j_{i}}}^{m+1} X_{-}^{\alpha_{k}} \prod_{\ell=i-p+1}^{i} \Delta X^{\alpha_{j_{\ell}}}\right)\right. \\
\cdots A_{\infty}^{\left.\alpha_{j_{1}}, \ldots, \alpha_{j_{i}-p-1} \mid \alpha_{j_{i-p}}\right]} \\
=\sum_{p=0}^{m-1} \sum_{i=p+2}^{m+1} \sum_{1 \leqslant j_{1}<\cdots<j_{i} \leqslant m+1} \mathbb{E}\left[Y_{\infty} \prod_{\substack{k=1 \\
k \neq j_{1}, \ldots, j_{i} \\
\alpha_{j_{1}}, \ldots, \alpha_{j_{i-p-1}} \mid \alpha_{j_{i-p}}}}^{m+1} X_{-}^{\alpha_{k}} \prod_{\ell=i-p+1}^{i} \Delta X^{\alpha_{j_{\ell}}}\right] \\
\cdots A_{\infty}
\end{gathered}
$$

Let us fix $p$ and $i, 0 \leqslant p \leqslant m-1, p+2 \leqslant i \leqslant m+1$, and $1 \leqslant j_{1}<\cdots<j_{i} \leqslant$ $m+1$. We consider the generic element

$$
K:=\left(\prod_{\substack{k=1 \\ k \neq j_{1}, \ldots, j_{i}}}^{m+1} X_{-}^{\alpha_{k}}\right)\left(\prod_{\ell=i-p+1}^{i} \Delta X^{\alpha_{j_{\ell}}}\right) .
$$

After expanding the product we observe that $K_{t}$ is equal to a finite sum of terms of type

$$
\prod_{k=1}^{q} X_{t-}^{\alpha_{i_{k}}} \prod_{j=q+1}^{q+r} X_{t}^{\alpha_{i_{j}}}, \quad 1 \leqslant i_{1}, \ldots, i_{q+r} \leqslant m+1 \text { pairwise different, } q+r \leqslant m-1 .
$$

The induction hypothesis, (28), and Lemma 3.3 yield $\mathbb{E}\left[Y_{\infty} K_{t}\right]=0$, $t \geqslant 0$. Thus (31) vanishes. This, together with (30) and (29), yields $\mathbb{E}\left[Y_{\infty} \prod_{i=1}^{m+1} X_{\infty}^{\alpha_{i}}\right]=0$. Hence, the proof of the induction step is finished and we can conclude that if $Y \perp \mathscr{X}$, then $\mathbb{E}\left[Y_{\infty} \prod_{i=1}^{m} X_{\infty}^{\alpha_{i}}\right]=0$ for every $m \in \mathbb{N}$ and $\alpha_{1}, \ldots, \alpha_{m} \in I$, which implies

$$
\mathbb{E}\left[Y_{\infty} \prod_{i=1}^{m} X_{t_{i}}^{\alpha_{i}}\right]=0, \quad t_{1}, \ldots, t_{m} \in \mathbb{R}_{+}, \quad \alpha_{1}, \ldots, \alpha_{m} \in \mathbb{R}_{+}, \quad m \in \mathbb{N},
$$

because the family $\mathscr{X}$ is assumed to be stable under stopping with respect to finite-valued deterministic stopping times. Therefore, if $Y$ is orthogonal to $\mathscr{X}$, then $Y_{\infty}$ is orthogonal in $L^{2}\left(\Omega, \mathscr{F} \mathscr{X}_{\infty}, \mathbb{P}\right)$ to $\mathscr{K}$, where $\mathscr{K}$ is the family of monomials defined in (25). But, by assumption, $\mathscr{K}$ is a total system in $L^{2}(\Omega, \mathscr{F} \mathscr{X}, \mathbb{P})$ and so $Y_{\infty}=0$. Thus $Y$ is indistinguishable from the process identically equal to zero. We have proven that, if $Y \in \mathscr{H}^{2}\left(\mathbb{F}^{\mathscr{X}}\right)$ is orthogonal to $\mathscr{X}$, then $Y$ is evanescent, i.e., that $\mathscr{X}$ satisfies condition $C_{2}$ which, from Proposition 2.1, is equivalent to the PRP of the family $\mathscr{X}$ with respect to $\mathbb{F}^{\mathscr{X}}$ and this completes the proof of Theorem 3.2. 
Now we consider some first examples of compensated-covariation stable families fulfilling all the assumptions of Theorem 3.2 and hence possessing the PRP.

Continuous Gaussian families. We start by considering $\mathscr{X}=\{W\}$, where $W$ is a (continuous) Wiener process with variance function $\sigma^{2}(t)=$ $\mathbb{E}\left[W_{t}^{2}\right], t \geqslant 0$ (cf. $\left[14\right.$, Definition I.4.9]), on the probability space $\left(\Omega, \mathscr{F}_{\infty}^{W}, \mathbb{P}\right)$, relative to the filtration $\mathbb{F}^{W}$. We observe that the random variable $W_{t}$ admits finite exponential moments of every order, for every $t \geqslant 0$, and hence the family $\mathscr{K}$ of monomials defined as in $(25)$ is total in $L^{2}\left(\Omega, \mathscr{F}_{\infty}^{W}, \mathbb{P}\right)$. Obviously, $[W, W]-\langle W, W\rangle=0$ and so the family $\mathscr{X} \cup\{0\}$ is compensatedcovariation stable satisfying all the assumptions of Theorem 3.2. Therefore $\mathscr{L}^{2}(W)=\mathscr{H}^{2}\left(\mathbb{F}^{\mathscr{X}}\right)=\mathscr{H}^{2}\left(\mathbb{F}^{W}\right)$. In other words, from Theorem 3.2, we can deduce the well-known fact (cf. [2]) that the Wiener process $W$ possesses the PRP with respect to $\mathbb{F}^{W}$.

More generally, on a complete probability space $(\Omega, \mathscr{F}, \mathbb{P})$ and with respect to a filtration $\mathbb{F}$ satisfying the usual conditions, we consider a family $\mathscr{X}:=\left\{X^{\alpha}, \alpha \in I\right\}$ of continuous local martingales such that $X_{0}^{\alpha}=0$ and $\left\langle X^{\alpha}, X^{\beta}\right\rangle$ is a deterministic (continuous) function, for every $\alpha$ and $\beta$ in $I$. We are going to show that such a family $\mathscr{X}$ of continuous local martingales is a Gaussian family with covariance function

$$
\operatorname{Cov}\left(X_{t}^{\alpha}, X_{s}^{\beta}\right)=\left\langle X^{\alpha}, X^{\beta}\right\rangle_{t \wedge s}, \quad s, t \geqslant 0 .
$$

The following result can be considered as a generalization of the theorem of P. Lévy on the characterization of the Wiener process (cf. [14, Theorem II.4.4]).

Proposition 3.2. A family of continuous local martingales $\mathscr{X}:=$ $\left\{X^{\alpha}, \alpha \in I\right\}$ such that $X_{0}^{\alpha}=0, \alpha \in I$, is a pairwise Gaussian family if and only if $\left\langle X^{\alpha}, X^{\beta}\right\rangle$ is deterministic, for every $\alpha$ and $\beta$ belonging to $I$. If one (and therefore both) of these conditions is satisfied, then $\mathscr{X}$ is Gaussian with covariance structure (32).

P r o o f. If $\mathscr{X}$ is a pairwise Gaussian family of continuous local martingale, then also the processes $X^{\alpha}+X^{\beta}$ and $X^{\alpha}-X^{\beta}$ are Gaussian continuous local martingales and therefore they have independent increments, for all $\alpha, \beta \in I$. This implies that $\left\langle X^{\alpha}+X^{\beta}, X^{\alpha}+X^{\beta}\right\rangle$ and $\left\langle X^{\alpha}-X^{\beta}, X^{\alpha}-X^{\beta}\right\rangle$ are deterministic and this together with the relation $\left\langle X^{\alpha}, X^{\beta}\right\rangle=\left(\left\langle X^{\alpha}+X^{\beta}, X^{\alpha}+X^{\beta}\right\rangle-\left\langle X^{\alpha}-X^{\beta}, X^{\alpha}-X^{\beta}\right\rangle\right) / 4$ implies that $\left\langle X^{\alpha}, X^{\beta}\right\rangle$ is deterministic, $\alpha, \beta \in I$. We now show the converse implication. It is enough to verify that for every $0<t_{1}<\cdots<t_{n}, n \in \mathbb{N}$, the vector $\left(X_{t_{1}}^{\alpha_{1}}, \ldots, X_{t_{n}}^{\alpha_{1}} ; \ldots ; X_{t_{1}}^{\alpha_{m}}, \ldots, X_{t_{n}}^{\alpha_{m}}\right)$ is Gaussian with the following covariance structure:

$$
\operatorname{Cov}\left(X_{t_{i}}^{\alpha_{j}}, X_{t_{\ell}}^{\alpha_{k}}\right)=\left\langle X^{\alpha_{j}}, X^{\alpha_{k}}\right\rangle_{t_{i} \wedge t_{\ell}}, \quad j, k=1, \ldots, m, \quad i, \ell=1, \ldots, n .
$$


For this it is sufficient to check that for every $t \geqslant 0$ the random vector $\left(X_{t}^{\alpha_{1}}, \ldots, X_{t}^{\alpha_{m}}\right)$ is Gaussian and its covariance function is $\operatorname{Cov}\left(X_{t}^{\alpha}, X_{t}^{\beta}\right)=$ $\left\langle X^{\alpha_{\ell}}, X^{\alpha_{k}}\right\rangle_{t}$, for every $m \in \mathbb{N}$. Indeed, we can assume that the family $\mathscr{X}$ is stable under stopping with respect to deterministic stopping times. If this would not be the case, we can consider the family $\mathscr{X}^{\mathscr{S}}$ defined as in (11), where $\mathscr{S}=\mathbb{R}_{+}$is the collection of all deterministic stopping times. Defining $Z:=\left(Z_{t}\right)_{t \geqslant 0}$ by

$$
Z_{t}:=\exp \left(i \sum_{j=1}^{m} u_{j} X_{t}^{\alpha_{j}}+\frac{1}{2} \sum_{\ell, k=1}^{m} u_{\ell} u_{k}\left\langle X^{\alpha_{\ell}}, X^{\alpha_{k}}\right\rangle_{t}\right)
$$

where $t \geqslant 0, u_{1}, \ldots, u_{m} \in \mathbb{R}$, and applying Itô's formula we get $Z_{t}=1+$ $\sum_{j=1}^{m} i u_{j} Z \cdot X_{t}^{\alpha_{j}}, t \geqslant 0$. Hence the process $Z$ is a (complex-valued) local martingale. But because $Z$ is bounded over compact intervals, it is in fact a true martingale, and we obtain $\mathbb{E}\left[Z_{t}\right]=\mathbb{E}\left[Z_{0}\right]=1$, which yields

$$
\mathbb{E}\left[\exp \left(i \sum_{j=1}^{m} u_{j} X_{t}^{\alpha_{j}}\right)\right]=\exp \left(-\frac{1}{2} \sum_{\ell, k=1}^{m} u_{\ell} u_{k}\left\langle X^{\alpha_{\ell}}, X^{\alpha_{k}}\right\rangle_{t}\right), \quad t \geqslant 0
$$

for all $u_{1}, \ldots, u_{m} \in \mathbb{R}$. In particular, this implies that the vector $\left(X_{t}^{\alpha_{1}}, \ldots, X_{t}^{\alpha_{m}}\right)$ is Gaussian with covariance function $\operatorname{Cov}\left(X_{t}^{\alpha}, X_{t}^{\beta}\right)=$ $\left\langle X^{\alpha_{\ell}}, X^{\alpha_{k}}\right\rangle_{t}$, for every $t \geqslant 0$, and the proof is finished. Proposition 3.2 is proved.

As a result of Proposition 3.2, a pairwise Gaussian family of continuous local martingales is always a Gaussian family of true martingales.

Let $\mathscr{X}:=\left\{X^{\alpha}, \alpha \in I\right\}$ be a family of continuous local martingales such that the process $\left\langle X^{\alpha}, X^{\beta}\right\rangle$ is deterministic, for every $\alpha, \beta \in I$, i.e., a pairwise Gaussian family of continuous local martingales. We restrict our attention to the probability space $\left(\Omega, \mathscr{F}_{\infty}^{\mathscr{X}}, \mathbb{P}\right)$ and to the filtration $\mathbb{F}^{\mathscr{X}}$. For every $\alpha \in I$ and for every $t \geqslant 0$, the random variable $X_{t}^{\alpha}$ admits finite exponential moments of every order. Therefore, the family $\mathscr{K}$ of monomials obtained from $\mathscr{X}$ is total in $L^{2}\left(\Omega, \mathscr{F}_{\infty} \mathscr{X}, \mathbb{P}\right)$. Thanks to the continuity of $X^{\alpha}$, $\alpha \in I$, we have that, for every $\alpha, \beta \in I$ the compensated-covariation process $X^{\alpha, \beta}$ is identically equal to zero and therefore $\mathscr{X} \cup\{0\}$ is a compensated covariation-stable family which satisfies all the assumptions of Theorem 3.2. This implies that $\mathscr{X}$ has the PRP with respect to $\mathbb{F}^{\mathscr{X}}$. For finite families $\mathscr{X}$ this result is due to Galtchouk [9]. If $\mathscr{X}$ is countable, say $\mathscr{X}:=\left\{X^{n}, n \geqslant 1\right\}$, and consists of orthogonal (i.e., independent) martingales, then it possesses the PRP relative to $\mathbb{F}^{\mathscr{X}}$ and (6) holds.

Independent families of Poisson processes. In [16] and [17] it was proved that a compensated Poisson process $X$ possesses the PRP with respect to $\mathbb{F}^{X}$. We now verify once again this result as a consequence of Theorem 3.2. We consider a Poisson process $N$ with general continuous intensity function $a(\cdot)$ (cf. [14, Definition I.3.26]) on the probability space 
$\left(\Omega, \mathscr{F}_{\infty}^{N}, \mathbb{P}\right)$. We define the family $\mathscr{X}$ by $\mathscr{X}:=\{X\}$, where $X:=N-a(\cdot)$ is the compensated Poisson process. Then we have

$$
[X, X]-\langle X, X\rangle=\sum_{0<s \leqslant \cdot}\left(\Delta N_{s}\right)^{2}-a(\cdot)=\sum_{0<s \leqslant \cdot} \Delta N_{s}-a(\cdot)=X
$$

and thus $\mathscr{X}$ is compensated-covariation stable. Hence all the assumptions of Theorem 3.2 are satisfied and so we can conclude that the family $\mathscr{X}$ has the PRP with respect to the filtration $\mathbb{F}^{N}=\mathbb{F}^{X}$. More generally, for a family $\mathscr{X}:=\left\{X^{\alpha}, \alpha \in I\right\}$ of independent compensated Poisson processes, on the probability space $\left(\Omega, \mathscr{F}_{\infty} \mathscr{X}, \mathbb{P}\right)$ and with respect to the filtration $\mathbb{F}^{\mathscr{X}}$, we have that, for every $\alpha, \beta \in I$ such that $\alpha \neq \beta$, the process $\left[X^{\alpha}, X^{\beta}\right]$ is equal to zero. This implies that also the process $\left\langle X^{\alpha}, X^{\beta}\right\rangle$ is equal to zero, i.e., $\mathscr{X}$ is a family of orthogonal martingales. Furthermore every $X_{t}^{\alpha}$ has finite exponential moments of every order, for every $\alpha \in I, t \geqslant 0$. Therefore, thanks to Theorem 3.2, the compensated-covariation stable family $\mathscr{X} \cup\{0\}$ possesses the $\mathrm{PRP}$ with respect to $\mathbb{F}^{\mathscr{X}}$. If $\mathscr{X}$ is moreover a countable family, say $\mathscr{X}:=\left\{X^{n}, n \geqslant 1\right\}$, then (6) holds.

4. The PRP for Lévy processes. In this section, given a Lévy process $L$, we construct families of martingales possessing the PRP relative to the augmented filtration generated by $L$. We start with a brief recall on Lévy processes and Poisson random measures.

A càdlàg process $L$ on a probability space $(\Omega, \mathscr{F}, \mathbb{P})$ such that $L_{0}=0$ is called a Lévy process if it is stochastically continuous and has homogeneous and independent increments. Let $L$ be a Lévy process. By $\mathscr{F}_{\infty}^{L}$ we denote the completed $\sigma$-algebra generated by $L$, i.e., $\mathscr{F}_{\infty}^{L}:=\sigma\left(L_{t}, t \geqslant 0\right) \vee \mathscr{N}(\mathbb{P})$, where $\mathscr{N}(\mathbb{P})$ denotes the $\mathbb{P}$-null sets with respect to $\sigma\left(L_{t}, t \geqslant 0\right)$. The natural filtration of $L$ is denoted by $\mathbb{F}^{L}=\left(\mathscr{F}_{t}^{L}\right)_{t \geqslant 0}$, where we put $\mathscr{F}_{t}^{L}:=\sigma\left(L_{s}, 0 \leqslant\right.$ $s \leqslant t) \vee \mathscr{N}(\mathbb{P})$. It is well known that $\mathbb{F}^{L}$ is right-continuous (cf. [24]) and that $L_{t}-L_{s}$ is independent of $\mathscr{F}_{s}^{L}, 0 \leqslant s \leqslant t$. From now on, we restrict ourselves to the probability space $\left(\Omega, \mathscr{F}_{\infty}^{L}, \mathbb{P}\right)$ equipped with the filtration $\mathbb{F}^{L}$.

On $(E, \mathscr{B}(E)):=\left(\mathbb{R}_{+} \times \mathbb{R}, \mathscr{B}\left(\mathbb{R}_{+}\right) \otimes \mathscr{B}(\mathbb{R})\right)$, where $\mathscr{B}(\cdot)$ denotes the Borel $\sigma$-algebra, we introduce the random measure $\mathrm{M}$ (cf. [14, Definition I.1.3]) by

$$
\mathrm{M}(\omega, A):=\sum_{s \geqslant 0} 1_{\left\{\Delta L_{s}(\omega) \neq 0\right\}} 1_{A}\left(s, \Delta L_{s}(\omega)\right), \quad \omega \in \Omega, \quad A \in \mathscr{B}(E),
$$

which we call the jump measure of $L$. It is easy to verify that $M$ is a homogeneous Poisson random measure relative to the filtration $\mathbb{F}^{L}$, i.e., an integer-valued random measure (cf. [14, Definition II.1.13]) such that (i) for every Borel subset $A \in \mathscr{B}(E), \mathbb{E}[\mathrm{M}(A)]=\left(\lambda_{+} \otimes \nu\right)(A)$, where $\lambda_{+}$is the Lebesgue measure on $\mathbb{R}_{+}$and $\nu$ is a $\sigma$-additive measure on $\mathbb{R}$; (ii) for all $s \geqslant 0$ and $A \in \mathscr{B}(E)$ such that $A \subseteq(s,+\infty) \times \mathbb{R}$, the random variable $\mathrm{M}(A)$ 
is independent of $\mathscr{F}_{s}^{L}$ (cf. [14, Definition II.1.20]). The $\sigma$-finite measure $\nu$ is the Lévy measure of $L$, which satisfies $\nu(\{0\})=0$ and $x^{2} \wedge 1 \in L^{1}(\nu)$. We put $\mathrm{m}:=\lambda_{+} \otimes \nu$.

Now we introduce the compensated Poisson random measure associated with the jump measure of $L$. The system $\mathscr{E}:=\{A \in \mathscr{B}(E): \mathrm{m}(A)<+\infty\}$ is a ring of Borel subsets of $E$. For every $A \in \mathscr{E}$ we define $\overline{\mathrm{M}}(A):=\mathrm{M}(A)-$ $\mathrm{m}(A)$. The family $\overline{\mathrm{M}}:=\{\overline{\mathrm{M}}(A), A \in \mathscr{E}\}$ is an orthogonal random measure (cf. [10, Chap. IV, §4]), i.e., for every $A, B \in \mathscr{E}, \overline{\mathrm{M}}(A) \in L^{2}(\mathbb{P})$,

$$
\mathbb{E}[\overline{\mathrm{M}}(A) \overline{\mathrm{M}}(B)]=\mathrm{m}(A \cap B)
$$

and if, moreover, $A \cap B=\varnothing$, then it follows

$$
\overline{\mathrm{M}}(A \cup B)=\overline{\mathrm{M}}(A)+\overline{\mathrm{M}}(B) .
$$

We call $\overline{\mathrm{M}}$ the compensated Poisson random measure (associated with $\mathrm{M}$ ).

Further we introduce the stochastic integral with respect to the jump measure $\mathrm{M}$ and the associated compensated Poisson random measure $\overline{\mathrm{M}}$ for measurable functions $f$ on $(E, \mathscr{B}(E))$. First we set $\mathrm{m}(f):=$ $\int_{E} f(t, x) \mathrm{m}(\mathrm{d} t, \mathrm{~d} x)$ if the integral on the right-hand side exists. If $f \geqslant 0$, $\mathrm{m}(f)$ is always well defined. Analogously, we can define the integral of $f$ with respect to $\mathrm{M} \omega$-wise. Under the assumption that $\int_{E}|f(t, x)| \mathrm{M}(\mathrm{d} t, \mathrm{~d} x)<+\infty$ a.s. holds, we define $\mathrm{M}(f):=\int_{E} f(t, x) \mathrm{M}(\mathrm{d} t, \mathrm{~d} x)$ a.s. and call $\mathrm{M}(f)$ the stochastic integral of $f$ with respect to M. From [18, Lemma $12.13(\mathrm{i})]$, we know that $\mathrm{M}(f)$ exists and is finite a.s. if and only if $\mathrm{m}(|f| \wedge 1)<\infty$. For $f \in L^{1}(\mathrm{~m})$ we have $\mathbb{E}[\mathrm{M}(f)]=\mathrm{m}(f)$.

The stochastic integral with respect to $\bar{M}$ for deterministic functions in $L^{2}(\mathrm{~m})$ is defined as in [10, Chap. IV], and we do not repeat the definition in detail. We only recall that in a first step the stochastic integral with respect to $\overline{\mathrm{M}}$ is defined for simple functions in $L^{2}(\mathrm{~m})$ and then is extended by isometry and denseness to arbitrary functions in $L^{2}(\mathrm{~m})$ : There exists a unique isometric mapping on $L^{2}(\mathrm{~m})$ into $L^{2}(\mathbb{P})$, again denoted by $\overline{\mathrm{M}}$, such that $\overline{\mathrm{M}}\left(1_{B}\right)=\overline{\mathrm{M}}(B), B \in \mathscr{E}$. If $f \in L^{2}(\mathrm{~m})$, then $\overline{\mathrm{M}}(f)$ is called the stochastic integral of $f$ with respect to the compensated Poisson random measure $\overline{\mathrm{M}}$. The following proposition holds.

Proposition 4.1. If $f \in L^{1}(\mathrm{~m}) \cap L^{2}(\mathrm{~m})$, then $\overline{\mathrm{M}}(f)=\mathrm{M}(f)-\mathrm{m}(f)$.

For any $f \in L^{2}(\nu)$ we introduce the process $X^{f}=\left(X_{t}^{f}\right)_{t \geqslant 0}$ by

$$
X_{t}^{f}=\overline{\mathrm{M}}\left(1_{[0, t]} f\right), \quad t \geqslant 0 .
$$

We use the notation $\nu(f):=\int_{\mathbb{R}} f(x) \nu(\mathrm{d} x)$, if the integral on the right-hand side exists.

Theorem 4.1. For every $f \in L^{2}(\nu)$ the process $X^{f}$ defined by (33) has the following properties: 
(i) $\left(X^{f}, \mathbb{F}^{L}\right)$ is a Lévy process and $\mathbb{E}\left[\left(X_{t}^{f}\right)^{2}\right]=t \nu\left(f^{2}\right)<+\infty$;

(ii) $X^{f} \in \mathscr{H}_{\mathrm{loc}}^{2}\left(\mathbb{F}^{L}\right)$ and $\left\langle X^{f}, X^{g}\right\rangle_{t}=t \nu(f g), f, g \in L^{2}(\nu)$; for every $T \in \mathbb{R}_{+}$, the stopped process $\left(X^{f}\right)^{T}$ belongs to $\mathscr{H}^{2}\left(\mathbb{F}^{L}\right)$;

(iii) $\Delta X^{f}=f(\Delta L) 1_{\{\Delta L \neq 0\}}$ a.s. and $X^{f}$ is locally bounded if $f$ is bounded;

(iv) $X^{f}=0$ a.s. if and only if $f=0 \nu$-a.e.;

(v) $X^{f}$ and $X^{g}$ are orthogonal if and only if $f, g \in L^{2}(\nu)$ are orthogonal.

The Itô-Lévy decomposition of $L$ asserts that there exists a Wiener process relative to $\mathbb{F}$ with variance function $\sigma^{2}(t):=\sigma^{2} t$ (cf. [14, Definition I.4.9]), say $\mathrm{W}^{\sigma}$, such that the following decomposition holds:

$$
L_{t}=\beta t+\mathrm{W}_{t}^{\sigma}+\mathrm{M}\left(1_{[0, t] \times\{|x|>1\}} x\right)+\overline{\mathrm{M}}\left(1_{[0, t] \times\{|x| \leqslant 1\}} x\right), \quad t \geqslant 0, \quad \text { a.s. },
$$

where $\beta \in \mathbb{R}$. We call the triplet $\left(\beta, \sigma^{2}, \nu\right)$ and the process $\mathrm{W}^{\sigma}$ the characteristics and the Gaussian part of $L$, respectively.

4.1. Compensated-covariation stable families of martingales. In this subsection, given a Lévy process $L$ with characteristics $\left(\beta, \sigma^{2}, \nu\right)$, our aim is to construct compensated-covariation stable families of martingales relative to $\mathbb{F}^{L}$ possessing the PRP.

Let $\left(L, \mathbb{F}^{L}\right)$ be a Lévy process with Gaussian part $\mathrm{W}^{\sigma}$ and jump measure M. By $\mathbb{F}^{\mathrm{W}^{\sigma}}$ we denote the completion in $\mathscr{F}_{\infty}^{L}$ of the filtration generated by $\mathrm{W}^{\sigma}$. We introduce the $\sigma$-algebra $\mathscr{F}_{t}^{\mathrm{M}}:=\sigma(\{\mathrm{M}(A), A \in \mathscr{B}(E), A \subseteq$ $[0, t] \times \mathbb{R}\}) \vee \mathscr{N}(\mathbb{P})$, for every $t \in[0,+\infty]$, where $\mathscr{N}(\mathbb{P})$ denotes the system of the $\mathbb{P}$-null sets of $\mathscr{F}_{\infty}^{L}$, and put $\mathbb{F}^{\mathrm{M}}=\left(\mathscr{F}_{t}^{\mathrm{M}}\right)_{t \geqslant 0}$. It is easy to see that $\mathbb{F}^{L}=\mathbb{F}^{\mathrm{W}^{\sigma}} \vee \mathbb{F}^{\mathrm{M}}$ and $\mathscr{F}_{\infty}^{L}=\mathscr{F}_{\infty}^{\mathrm{W}^{\sigma}} \vee \mathscr{F}_{\infty}^{\mathrm{M}}$.

In the following we shall consider a system $\mathscr{C}$ of real-valued measurable functions on $\mathbb{R}$. We make the following assumption.

Assumption 4.1. (i) $\mathscr{C} \subseteq L^{1}(\nu) \cap L^{2}(\nu)$;

(ii) $\mathscr{C}$ is total in $L^{2}(\nu)$;

(iii) $\mathscr{C}$ is stable under multiplication;

(iv) $\mathscr{C}$ is a system of bounded functions.

We observe that, given a Lévy measure $\nu$, a system $\mathscr{C}$ satisfying Assumption 4.1 always exists: For example, $\mathscr{C}:=\left\{1_{(a, b]}, a, b \in \mathbb{R}: a<b, 0 \notin\right.$ $[a, b]\} \cup\{0\}$ is such a system.

For a system $\mathscr{C}$ satisfying Assumption 4.1, we now define the family $\mathscr{X}_{\mathscr{C}}$ as follows:

$$
\mathscr{X}_{\mathscr{C}}:=\left\{\mathrm{W}^{\sigma}\right\} \cup\left\{X^{f}, f \in \mathscr{C}\right\}
$$

where $X^{f}, f \in L^{2}(\nu)$, was introduced in (33). Then the following proposition holds.

Proposition 4.2. The family of $\mathbb{F}^{L}$-martingales $\mathscr{X}_{\mathscr{C}} \cup\{0\} \subseteq \mathscr{H}_{\mathrm{loc}}^{2}$, where $\mathscr{X}_{\mathscr{C}}$ is defined in (35), is compensated-covariation stable. Moreover $\mathscr{X}_{\mathscr{C}}$ generates the filtration $\mathbb{F}^{L}$ and the $\sigma$-algebra $\mathscr{F}_{\infty}^{L} ;$ for every $X \in \mathscr{X}_{\mathscr{C}}$, 
$\mathbb{E}\left[\exp \left(c\left|X_{t}\right|\right)\right]<+\infty$, for all $c>0, t \geqslant 0$, and $\langle X, Y\rangle$ is deterministic, for all $X, Y \in \mathscr{X}_{\mathscr{C}}$.

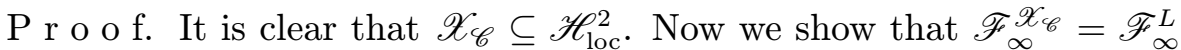
$\left(=\mathscr{F}_{\infty}^{\mathrm{W}^{\sigma}} \vee \mathscr{F}_{\infty}^{\mathrm{M}}\right)$. The proof that the family $\mathscr{X}_{\mathscr{C}}$ generates the filtration $\mathbb{F}^{L}$ is analogous and will be omitted. Proposition 4.1 yields that for every $f \in \mathscr{C} \subseteq$ $L^{1}(\nu) \cap L^{2}(\nu)$ and $t \geqslant 0$ the equality $X_{t}^{f}=\mathrm{M}\left(1_{[0, t]} f\right)-t \nu(f)$ holds. Hence, introducing the $\sigma$-field $\mathscr{G}:=\sigma\left(\left\{\mathrm{M}\left(1_{[0, t]} f\right), 0 \leqslant t<\infty, f \in \mathscr{C}\right\}\right)$, it suffices to show that the equality $\mathscr{G} \vee \mathscr{N}(\mathbb{P})=\mathscr{F}_{\infty}^{\mathrm{M}}$ is fulfilled. Obviously $\mathrm{M}(f)$ is $\mathscr{F}_{\infty}^{\mathrm{M}}$ measurable for every nonnegative measurable function $f$. Since $\mathscr{C} \subseteq L^{1}(\nu)$, for every $f \in \mathscr{C}$ and $t \geqslant 0$ we can write $\mathrm{M}\left(1_{[0, t]} f\right)=\mathrm{M}\left(1_{[0, t]} f^{+}\right)-\mathrm{M}\left(1_{[0, t]} f^{-}\right)$ a.s. which is $\mathscr{F}_{\infty}^{\mathrm{M}}$-measurable. This yields the inclusion $\mathscr{G} \vee \mathscr{N}(\mathbb{P}) \subseteq \mathscr{F}_{\infty}^{M}$. Conversely, let $B_{n} \subseteq \mathbb{R}$ be such that $B_{n} \uparrow \mathbb{R}$ and $\nu\left(B_{n}\right)<+\infty$ for all $n \geqslant 1$. Using the monotone class theorem we deduce that $\mathrm{M}\left(1_{[0, t] \times B_{n}} g\right)$ is $\mathscr{G} \vee \mathscr{N}(\mathbb{P})$ measurable for every bounded measurable function $g$ on $E=\mathbb{R}_{+} \times \mathbb{R}$ and hence for $g=1_{A}$ with $A \in \mathscr{B}(E)$. Finally, letting $n \rightarrow \infty$, we observe that $\mathrm{M}(([0, t] \times \mathbb{R}) \cap A)$ is $\mathscr{G} \vee \mathscr{N}(\mathbb{P})$-measurable for all $A \in \mathscr{B}(E)$, proving the inclusion $\mathscr{F}_{\infty}^{\mathrm{M}} \subseteq \mathscr{G} \vee \mathscr{N}(\mathbb{P})$. The family $\mathscr{K}$ of monomials generated by $\mathscr{X}_{\mathscr{C}}$ is total in $L^{2}\left(\Omega, \mathscr{F}_{T}^{\mathscr{X}_{\mathscr{C}}}, \mathbb{P}\right)$, and from the previous step, $\mathscr{F}_{T}^{\mathscr{X}_{\mathscr{C}}}=\mathscr{F}_{T}^{L}$. Indeed, the Lévy measure of $X^{f}$ is $\nu^{f}$, where we put $\nu^{f}(\{0\}):=0$ and $\nu^{f}(B):=\nu \circ f^{-1}(B), B \in \mathscr{B}(\mathbb{R}), B \subseteq \mathbb{R} \backslash\{0\}$. Because each function $f$ in $\mathscr{C}$ is bounded, $\nu^{f}$ has bounded support. As a consequence of [22, Lemmas 25.6 and 27.7], it follows that $X_{t}^{f}$ has finite exponential moments of every order, $t \in[0, T], f \in \mathscr{C}$. The process $\mathrm{W}^{\sigma}$ is a Wiener process and hence $\mathrm{W}_{t}^{\sigma}$ has exponential moments of every order, $t \in[0, T]$, and the claim about the totality of monomials follows from Theorem 3.1. Obviously, we have $\left\langle\mathrm{W}^{\sigma}, \mathrm{W}^{\sigma}\right\rangle_{t}=\sigma^{2} t, t \geqslant 0$, and $\left\langle\mathrm{W}^{\sigma}, X^{f}\right\rangle=0$ for every $f \in \mathscr{C}$. Moreover, if $f, g \in \mathscr{C}$, then $\left\langle X^{f}, X^{g}\right\rangle_{t}=t \nu(f g)$ (cf. Theorem 4.1). Therefore, $\langle X, Y\rangle$ is a deterministic process, for every $X, Y \in \mathscr{X}_{\mathscr{C}}$. It remains to show that $\mathscr{X}_{\mathscr{C}} \cup\{0\}$ is a compensated-covariation stable family. For this we first notice that $\left[\mathrm{W}^{\sigma}, \mathrm{W}^{\sigma}\right]-\left\langle\mathrm{W}^{\sigma}, \mathrm{W}^{\sigma}\right\rangle=0$ and $\left[\mathrm{W}^{\sigma}, X^{f}\right]-\left\langle\mathrm{W}^{\sigma}, X^{f}\right\rangle=0, f \in \mathscr{C}$, and so these processes belong to $\mathscr{X}_{\mathscr{C}} \cup\{0\}$. Furthermore, for given $f, g \in \mathscr{C}$, using (8), (2) and Theorem 4.1 (ii), (iii), we can compute the compensatedcovariation process as

$$
\begin{aligned}
X_{t}^{f, g} & :=\left[X^{f}, X^{g}\right]_{t}-\left\langle X^{f}, X^{g}\right\rangle_{t} \\
& =\sum_{0<s \leqslant t} f\left(\Delta L_{s}\right) g\left(\Delta L_{s}\right) 1_{\left\{\Delta L_{s} \neq 0\right\}}-\nu(f g) t=X_{t}^{f g}, \quad t \geqslant 0, \quad \text { a.s. }
\end{aligned}
$$

where in the last equality we used that $f g \in \mathscr{C} \subseteq L^{1}(\nu) \cap L^{2}(\nu)$ and Proposition 4.1. Hence $X^{f, g}$ belongs to $\mathscr{X}_{\mathscr{C}}$. This proves that $\mathscr{X}_{\mathscr{C}} \cup\{0\}$ is a compensated-covariation stable family. Proposition 4.2 is proved.

The following result is a consequence of Proposition 4.2 and Theorem 3.2. 
Proposition 4.3. Let $\left(L, \mathbb{F}^{L}\right)$ be a Lévy process with characteristics $\left(\beta, \sigma^{2}, \nu\right)$ and $\mathscr{C}$ satisfy Assumption 4.1. Then the family $\mathscr{X}_{\mathscr{C}}$ defined in (35) possesses the PRP with respect to $\mathbb{F}^{L}$.

P r o o f. From Proposition 4.2 and Theorem 3.1, the family $\mathscr{X}_{\mathscr{C}} \cup\{0\}$ satisfies all the assumptions of Theorem 3.2 and therefore it possesses the PRP with respect to $\mathbb{F}_{+}^{\mathscr{X}_{\mathscr{C}} \cup\{0\}}$, which is equal to $\mathbb{F}^{L}$. Hence $\mathscr{L}^{2}\left(\mathscr{X}_{\mathscr{C}}\right)=$ $\mathscr{L}^{2}\left(\mathscr{X}_{\mathscr{C}} \cup\{0\}\right)=\mathscr{H}^{2}\left(\mathbb{F}^{L}\right)$, and the proof is concluded. Proposition 4.3 is proved.

4.2. Families of martingales with the PRP. In this subsection we want to extend the result of Proposition 4.3 to more general systems of functions in $L^{2}(\nu)$ : We now consider an arbitrary system $\mathscr{T} \subseteq L^{2}(\nu)$ and define the family $\mathscr{X}_{\mathscr{T}}$ by

$$
\mathscr{X}_{\mathscr{T}}:=\left\{\mathrm{W}^{\sigma}\right\} \cup\left\{X^{f}, f \in \mathscr{T}\right\} .
$$

In the following theorem we establish necessary and sufficient conditions on the system $\mathscr{T} \subseteq L^{2}(\nu)$ for the family $\mathscr{X}_{\mathscr{T}}$ to have the PRP with respect to $\mathbb{F}^{L}$.

Theorem 4.2. Let $\mathscr{T}$ be a system of functions in $L^{2}(\nu)$. The family $\mathscr{X}_{\mathscr{T}}$ defined in (36) possesses the PRP with respect to $\mathbb{F}^{L}$ if and only if $\mathscr{T}$ is a total system in $L^{2}(\nu)$, i.e., $\operatorname{Span}(\mathscr{T})$ is dense in $L^{2}(\nu)$.

P r o o f. First we assume that the family $\mathscr{X}_{\mathscr{T}}$ possesses the PRP and show that $\mathscr{T}$ is total in $L^{2}(\nu)$. If $\mathscr{X}_{\mathscr{T}}$ has the PRP, in view of Proposition 2.1, it satisfies condition $C_{2}$. We consider a function $h \in L^{2}(\nu)$ which is orthogonal to the closure in $L^{2}(\nu)$ of $\operatorname{Span}(\mathscr{T})$. By Theorem $4.1(\mathrm{v})$, the martingale $X^{h} \in \mathscr{H}_{\mathrm{loc}}^{2}$ associated with $h$ is orthogonal to $\mathscr{X}_{\mathscr{T}}$. But then, by condition $C_{2}$, we have $X^{h}=0$ and by Theorem 4.1 (iv), $h=0$ meaning, in particular, that $\mathscr{T}$ is total in $L^{2}(\nu)$. Conversely, we assume that $\mathscr{T}$ is total in $L^{2}(\nu)$ and we show that $\mathscr{X}_{\mathscr{T}}$ has the PRP with respect to $\mathbb{F}^{L}$. For this aim we consider a system $\mathscr{C}$ satisfying Assumption 4.1. From Proposition 4.3 we know that $\mathscr{X}_{\mathscr{C}}$ has the PRP with respect to $\mathbb{F}^{L}$. Hence it is enough to prove that $\mathscr{L}^{2}\left(\mathscr{X}_{\mathscr{C}}\right) \subseteq \mathscr{L}^{2}\left(\mathscr{X}_{\mathscr{T}}\right)$. For $N \in \mathbb{N}$, we put $\mathscr{X}_{\mathscr{C}}^{N}:=\left\{\left(\mathrm{W}^{\sigma}\right)^{N}\right\} \cup\left\{\left(X^{f}\right)^{N}, f \in \mathscr{C}\right\}$, where the superscript $N$ denotes the operation of stopping at the deterministic time $N \in \mathbb{N} ; \mathscr{X}_{\mathscr{T}}^{N}$ is introduced from $\mathscr{X}_{\mathscr{T}}$ analogously. Because $\mathscr{T}$ is total in $L^{2}(\nu)$, for every $f \in \mathscr{C}$ there exists a sequence $\left(f_{n}\right)_{n \in \mathbb{N}} \subseteq \operatorname{Span}(\mathscr{T})$ such that $f_{n} \rightarrow f$ in $L^{2}(\nu)$ as $n \rightarrow+\infty$. From the linearity of $X^{g}$ with respect to $g \in L^{2}(\nu)$, we conclude that $X^{f_{n}}$ belongs to the linear hull of $\mathscr{X}_{\mathscr{T}}$. The processes $\left(X^{f}\right)^{N}$ and $\left(X^{f_{n}}\right)^{N}$ belong to $\mathscr{H}^{2}$ for every $N \in \mathbb{N}$ (cf. Theorem 4.1 (ii)). By isometry, for any $N \in \mathbb{N}$, we get

$$
\left\|\left(X^{f}\right)^{N}-\left(X^{f_{n}}\right)^{N}\right\|_{\mathscr{H}^{2}}=\left\|X_{N}^{f}-X_{N}^{f_{n}}\right\|_{L^{2}(\mathbb{P})}=\left\|X_{N}^{f-f_{n}}\right\|_{L^{2}(\mathbb{P})}=\sqrt{N}\left\|f-f_{n}\right\|_{L^{2}(\nu)}
$$


which converges to zero as $n \rightarrow+\infty$. This implies that $\mathscr{X}_{\mathscr{C}}^{N}$ is contained in the closure in $\left(\mathscr{H}^{2},\|\cdot\|_{\mathscr{H}^{2}}\right)$ of the linear hull of $\mathscr{X}_{\mathscr{T}}^{N}$ and hence, by stability, in $\mathscr{L}^{2}\left(\mathscr{X}_{\mathscr{T}}\right)$. The sequence $(N)_{N=1,2, \ldots}$ localizes $X^{f}$ to $\mathscr{H}^{2}$ and hence $\mathscr{L}^{2}\left(\left\{X^{f^{N}}, N \in \mathbb{N}\right\}\right)=\mathscr{L}^{2}\left(X^{f}\right)$. As a consequence, the stability of $\mathscr{L}^{2}\left(\mathscr{X}_{\mathscr{T}}\right)$ and Theorem 2.1 imply $\mathscr{L}^{2}\left(\mathscr{X}_{\mathscr{C}}\right) \subseteq \mathscr{L}^{2}\left(\mathscr{X}_{\mathscr{T}}\right)$. Proposition 4.3 ensures $\mathscr{H}^{2}=\mathscr{L}^{2}\left(\mathscr{X}_{\mathscr{C}}\right)$, therefore from the previous inclusion we deduce $\mathscr{H}^{2}=\mathscr{L}^{2}\left(\mathscr{X}_{\mathscr{T}}\right)$ and the proof is finished. Theorem 4.2 is proved.

Now we turn to the problem of finding a finite family of martingales $\mathscr{X}_{\mathscr{T}}$ possessing the PRP with respect to $\mathbb{F}^{L}$. Although this is particularly important, especially for applications, this is possible only with a rather limited generality, as the following corollary states.

Corollary 4.1. Let $\left(L, \mathbb{F}^{L}\right)$ be a Lévy process with characteristics $\left(\beta, \sigma^{2}, \nu\right)$. The following statements are equivalent: (i) There exists a finite family $\mathscr{X}_{\mathscr{T}}$ possessing the PRP with respect to $\mathbb{F}^{L}$; (ii) $L^{2}(\nu)$ is finite dimensional; (iii) $\nu$ has finite support.

The situation discussed in Corollary 4.1 occurs if $L$ is a simple Lévy process, i.e., it is of the form $L_{t}:=\mathrm{W}_{t}^{\sigma}+\alpha_{1} N_{t}^{1}+\cdots+\alpha_{m} N_{t}^{m}, t \geqslant 0$, where $\left(\mathrm{W}^{\sigma}, \mathbb{F}^{L}\right)$ is a Brownian motion with variance function $\left\langle\mathrm{W}^{\sigma}, \mathrm{W}^{\sigma}\right\rangle_{t}=$ $\sigma^{2} t ;\left(N^{j}, \mathbb{F}^{L}\right)$ is a homogeneous Poisson process with parameter $\gamma_{j}>0$, $j=1, \ldots, m$, and $\left(\mathrm{W}^{\sigma}, N^{1}, \ldots, N^{m}\right)$ is an independent vector of processes; $\alpha_{1}, \ldots, \alpha_{m}$ are real numbers.

We now consider the important case in which the system $\mathscr{T} \subseteq L^{2}(\nu)$ is a complete orthogonal system, i.e., a total system consisting of orthogonal functions. In this case the system $\mathscr{T}$ is countable and we use the notation $\mathscr{T}:=\left\{f_{n}, n \geqslant 1\right\}$. Under these conditions the PRP becomes particularly interesting. We stress that, because $L^{2}(\nu)$ is a separable Hilbert space, it is always possible to construct a complete orthogonal system.

Theorem 4.3. Let $\mathscr{T}$ be a complete orthogonal system in $L^{2}(\nu)$. Then the associated family $\mathscr{X}_{\mathscr{T}}$ has the PRP and any martingale $X$ belonging to $\mathscr{H}^{2}$ can be represented as

$$
X=\phi \cdot \mathrm{W}^{\sigma}+\sum_{n=1}^{\infty} H^{n} \cdot X^{f_{n}}, \quad \phi \in \mathrm{L}^{2}\left(\mathrm{~W}^{\sigma}\right), \quad H^{n} \in \mathrm{L}^{2}\left(X^{f_{n}}\right), \quad n \geqslant 1,
$$

where the series converges in $\left(\mathscr{H}^{2},\|\cdot\|_{\mathscr{H}^{2}}\right)$.

P r o o f. Because of the orthogonality of $\mathscr{T}$, the associated family of martingales $\mathscr{X}_{\mathscr{T}}$ is orthogonal (cf. Theorem $\left.4.1(\mathrm{v})\right)$. We have $\mathscr{L}^{2}\left(\mathscr{X}_{\mathscr{T}}\right)=$ $\mathscr{H}^{2}$ thanks to Theorem 4.2, and the assertion follows from (6). Theorem 4.3 is proved.

$\mathrm{R}$ e $\mathrm{m}$ a r k 4.1. It is well known that any martingale $X \in \mathscr{H}^{2}\left(\mathbb{F}^{L}\right)$ has a representation

$$
X=H \cdot \mathrm{W}^{\sigma}+G * \overline{\mathrm{M}},
$$


where $H \in \mathrm{L}^{2}\left(\mathrm{~W}^{\sigma}\right)$ and $G \in \mathrm{G}^{2}(\mathrm{M})$ (for the definition of $\mathrm{G}^{2}(\mathrm{M})$ and of the stochastic integral $G * \overline{\mathrm{M}}$, see [13], formula (3.62) and Definition 3.63, respectively). This integral representation is due to Kunita and Watanabe [20]. Also see [14, Theorem III.4.34], where the proof is based on the uniqueness of the solution of a certain martingale problem. Another approach is given by Kunita [19] using the chaos expansion of the seminal paper by Itô [12].

Here we want to present a sketch of an alternative and independent proof of the integral representation (38) using Theorem 4.3. Let $\mathscr{T}=\left\{f_{n}, n \geqslant 1\right\}$ be a complete orthogonal system of $L^{2}(\nu)$ and $\mathscr{X}_{\mathscr{T}}$ the associated family of martingales. By Theorem 4.3 each martingale $X \in \mathscr{H}^{2}\left(\mathbb{F}^{L}\right)$ can be represented as in (37). Let $\left(H^{n}\right)_{n \geqslant 1}$ be the sequence of integrands appearing in (37). Because $H^{n} f_{n} \in \mathrm{G}^{2}(\mathrm{M})$, we have $H^{n} \cdot X^{f_{n}}=H^{n} f_{n} * \overline{\mathrm{M}}$ (cf. [13, Definition 3.63]). So we can rewrite (37) as

$X=\phi \cdot \mathrm{W}^{\sigma}+\sum_{n=1}^{\infty}\left\{\left(H^{n} f_{n}\right) * \overline{\mathrm{M}}\right\}, \quad \phi \in \mathrm{L}^{2}\left(\mathrm{~W}^{\sigma}\right), \quad H^{n} \in \mathrm{L}^{2}\left(X^{f_{n}}\right), \quad n \geqslant 1$

It follows that $\sum_{n=1}^{\infty} H^{n} f_{n}=: G$ belongs to $\mathrm{G}^{2}(\mathrm{M})$. But this means that we can consider the integral $G * \overline{\mathrm{M}} \in \mathscr{H}^{2}$ and $\sum_{n=1}^{\infty}\left\{\left(H^{n} f_{n}\right) * \overline{\mathrm{M}}\right\}=G * \overline{\mathrm{M}}$. Therefore every martingale $X \in \mathscr{H}^{2}\left(\mathbb{F}^{L}\right)$ can be represented as in (38), where $G \in \mathrm{G}^{2}(\mathrm{M})$ is defined as above.

Now we consider the case in which $L$ is a square integrable Lévy process, meaning that the function $x$ belongs to $L^{2}(\nu)$. In this case we can introduce the process $\bar{L}=\left(\bar{L}_{t}\right)_{t \geqslant 0}$ by setting $\bar{L}_{t}:=L_{t}-\mathbb{E}\left[L_{t}\right], t \geqslant 0$. We want to prove an analogue of Theorem 4.2 for the family

$$
\overline{\mathscr{X}}_{\mathscr{T}}:=\{\bar{L}\} \cup\left\{X^{f}, f \in \mathscr{T}\right\}
$$

where $\mathscr{T} \subseteq L^{2}(\nu)$. Such a result seems of major interest because in many applications observations of $L$ are available rather than of $\mathrm{W}^{\sigma}$. We stress that now the situation is slightly different than in Theorem 4.2.

Theorem 4.4. Let $\left(L, \mathbb{F}^{L}\right)$ be a square integrable Lévy process with characteristic triplet $\left(\beta, \sigma^{2}, \nu\right)$. Then $\overline{\mathscr{X}}_{\mathscr{T}}$ defined by $(39)$ possesses the PRP and the function $x$ belongs to the closure in $L^{2}(\nu)$ of $\mathscr{T}$ if and only if $\mathscr{T} \subseteq L^{2}(\nu)$ is a total system.

P r o o f. We start by proving that if $\mathscr{T} \subseteq L^{2}(\nu)$ is total, then $\overline{\mathscr{X}} \mathscr{T}$ possesses the PRP. It is clear that if $\mathscr{T}$ is total in $L^{2}(\nu)$, then the function $x$ belongs to the closure in $L^{2}(\nu)$ of $\operatorname{Span}(\mathscr{T})$, because $L$ is a square integrable Lévy process. As usual, we denote by $\mathrm{W}^{\sigma}$ the Gaussian part of $L$. It is enough to prove that $\mathscr{L}^{2}\left(\mathrm{~W}^{\sigma}\right) \subseteq \mathscr{L}^{2}\left(\overline{\mathscr{X}}_{\mathscr{T}}\right)$. Indeed, if it is the case, we have $\mathscr{L}^{2}\left(\mathscr{X}_{\mathscr{T}}\right) \subseteq \mathscr{L}^{2}\left(\overline{\mathscr{X}}_{\mathscr{T}}\right)$, where $\mathscr{X}_{\mathscr{T}}$ was introduced in $(36)$, and Theorem 4.2 yields the result. The sequence $(N)_{N=1,2, \ldots}$ of deterministic stopping times localizes the processes $X^{g}$ and $\bar{L}$ to $\mathscr{H}^{2}$, for every $g \in L^{2}(\nu)$. 
In particular, this implies that $\bar{L}^{N}$ belongs to $\mathscr{L}^{2}\left(\overline{\mathscr{X}}_{\mathscr{T}}\right)$, for every $N \geqslant 1$. Because of the square integrability of $L$, the function $x$ belongs to $L^{2}(\nu)$. The system $\mathscr{T}$ is total in $L^{2}(\nu)$ and therefore there exists $\left(f_{n}\right)_{n \geqslant 1} \subseteq \operatorname{Span}(\mathscr{T})$ converging to $x$ in $L^{2}(\nu)$ as $n \rightarrow+\infty$. The martingales $\left(X^{f_{n}}\right)^{N}, n \geqslant 1$, belong to $\mathscr{L}^{2}\left(\overline{\mathscr{X}}_{\mathscr{T}}\right)$. Using the linearity of $X^{g}$ with respect to $g \in L^{2}(\nu)$ and the isometry, we obtain that $\left(X^{f_{n}}\right)^{N}$ converges to $\left(X^{x}\right)^{N}$ in $\mathscr{H}^{2}$, for every $N \geqslant 1$. Hence $\left(X^{x}\right)^{N} \in \mathscr{L}^{2}\left(\overline{\mathscr{X}}_{\mathscr{T}}\right)$, for every $N \geqslant 1$. Using the ItôLévy decomposition of $\bar{L}$, we can write $\mathrm{W}^{\sigma}=\bar{L}-X^{x}$ and this implies that the family $\left\{\left(\mathrm{W}^{\sigma}\right)^{N}, N \geqslant 1\right\}$ is contained in $\mathscr{L}^{2}\left(\overline{\mathscr{X}}_{\mathscr{T}}\right)$. Hence

$$
\mathscr{L}^{2}\left(\mathrm{~W}^{\sigma}\right)=\mathscr{L}^{2}\left(\left\{\left(\mathrm{~W}^{\sigma}\right)^{N}, N \geqslant 1\right\}\right) \subseteq \mathscr{L}^{2}\left(\overline{\mathscr{X}}_{\mathscr{T}}\right) .
$$

Consequently, $\mathscr{L}^{2}\left(\overline{\mathscr{X}}_{\mathscr{T}}\right)=\mathscr{H}^{2}$ and the first part of the proof is finished. Now we assume that $\mathscr{X}_{\mathscr{T}}$ possesses the PRP and that the function $x$ belongs to the closure in $L^{2}(\nu)$ of $\operatorname{Span}(\mathscr{T})$ and we prove that $\mathscr{T}$ is total in $L^{2}(\nu)$. Because $\overline{\mathscr{X}}_{\mathscr{T}}$ possesses the PRP, it satisfies condition $C_{2}$ (cf. Proposition 2.1). We consider $h \in L^{2}(\nu)$ such that $h$ is orthogonal to the closure of $\operatorname{Span}(\mathscr{T})$ in $L^{2}(\nu)$. This implies, in particular, that $h$ is orthogonal to $x$. Therefore, from $\bar{L}:=\mathrm{W}^{\sigma}+X^{x}$, we get that the martingale $X^{h} \in \mathscr{H}_{\text {loc }}^{2}$ is orthogonal to $\overline{\mathscr{X}}_{\mathscr{T}}$ (cf. Theorem $4.1(\mathrm{v})$ ). But then, from condition $C_{2}$, it follows $X^{h}=0$ which implies $h=0$ (cf. Theorem 4.1 (iv)), meaning that $\mathscr{T}$ is total in $L^{2}(\nu)$, and this completes the proof of Theorem 4.4.

We note that an analogue of Corollary 4.1 holds for $\overline{\mathscr{X}}_{\mathscr{T}}$. Finally, we remark the following: To an orthogonal system $\mathscr{T} \subseteq L^{2}(\nu)$ there corresponds a family of orthogonal martingales $\overline{\mathscr{X}}_{\mathscr{T}}$ if and only if $x$ is orthogonal to $\mathscr{T}$. Consequently, if we have chosen $\mathscr{T}$ such that $\overline{\mathscr{X}}_{\mathscr{T}}$ possesses the PRP, i.e., a total system of $L^{2}(\nu)$, then the family $\overline{\mathscr{X}}_{\mathscr{T}}$ is not orthogonal (because, by Theorem 4.4, the function $x$ belongs to the closure in $L^{2}(\nu)$ of $\left.\mathscr{T}\right)$. However, if $\mathscr{T}$ is a countable total family, we can obtain a family of orthogonal martingales possessing the PRP directly by orthogonalizing $\overline{\mathscr{X}}_{\mathscr{T}}$. We refer to, e.g., [20] for an analogue of the Gram-Schmidt orthogonalization procedure for locally square integrable martingales.

\subsection{Examples of families with the PRP relative to the Lévy filtration.}

Compensated Poisson processes. The first example that we discuss is elementary but important because it exhibits a concrete family of locally square integrable martingales with the PRP that can be introduced for any Lévy process $L$, without further assumptions on the Lévy measure $\nu$. Let $\left(L, \mathbb{F}^{L}\right)$ be a Lévy process with characteristic triplet $\left(\beta, \sigma^{2}, \nu\right)$. The system $\widetilde{T}_{D}:=\left\{1_{(a, b]}, 0 \notin[a, b], a, b \in D\right\}$, where $D \subseteq \mathbb{R}$ is a countable dense set as, e.g., the set of dyadic rational numbers, is total in $L^{2}(\nu)$. From Theorem 4.2 the family $\mathscr{X}_{\widetilde{T}_{D}}:=\left\{\mathrm{W}^{\sigma}\right\} \cup\left\{X^{f}, f \in \widetilde{\mathscr{T}}_{D}\right\}$ possesses the PRP with respect to $\mathbb{F}^{L}$. Of course $\widetilde{\mathscr{T}}_{D}$ is, in general, not orthogonal but it can be 
orthogonalized to a system, say $\mathscr{T}_{D}$, such that the family $\mathscr{X}_{\mathscr{T}_{D}}$ possesses the PRP with respect to $\mathbb{F}^{L}$ and every martingale $X \in \mathscr{H}^{2}$ can be represented as described in Theorem 4.3.

Teugels martingales. This example shows that under some assumptions on the Lévy measure $\nu$, Teugels martingales can be introduced as a family possessing the $\mathrm{PRP}$ with respect to $\mathbb{F}^{L}$. Teugels martingales were considered in [21], where, among other results, it was also proved that the orthogonalized Teugels martingales possess the PRP. We are going to obtain the PRP for the orthogonalized Teugels martingales as an application of the theory that we developed.

Let $\left(L, \mathbb{F}^{L}\right)$ be a Lévy process with characteristic triplet $\left(\beta, \sigma^{2}, \nu\right)$. We require that there exist two constants $\varepsilon, \lambda>0$ such that $\mathrm{e}^{\lambda|x| / 2} 1_{\{|x|>\varepsilon\}} \in$ $L^{2}(\nu)$. From this assumption it follows that the functions $x^{n}$ belong to $L^{1}(\nu) \cap L^{2}(\nu)$ for every $n \geqslant 2$ and $x$ belongs to $L^{2}(\nu)$. Moreover, for every fixed $m \geqslant 1$, the system $\left\{x^{n}, n \geqslant m\right\}$ is total in $L^{2}(\nu)$. Because of $x^{n} \in L^{2}(\nu)$, $n \geqslant 1$, we can introduce the martingales $X^{n}=\left(X_{t}^{n}\right)_{t \geqslant 0}$ as $X_{t}^{n}:=\overline{\mathrm{M}}\left(1_{[0, t]} x^{n}\right)$, $t \geqslant 0, n \geqslant 2$. We put $X^{1}:=\bar{L}$, where $\bar{L}_{t}:=L_{t}-\mathbb{E}\left[L_{t}\right], t \geqslant 0$. With the subsystem $\mathscr{T}:=\left\{x^{n}, n \geqslant 2\right\} \subseteq L^{2}(\nu)$, we associate the family of martingales given by $\overline{\mathscr{X}}_{\mathscr{T}}:=\{\bar{L}\} \cup\left\{X^{n}, n \geqslant 2\right\}=\left\{X^{n}, n \geqslant 1\right\}: \overline{\mathscr{X}}_{\mathscr{T}}$ is the family of Teugels martingales and, from Theorem 4.4, $\bar{X}_{\mathscr{T}}$ has the PRP with respect to $\mathbb{F}^{L}$. We stress that, because of the assumptions on the Lévy measure, the family of Teugels martingales is also a compensated-covariation stable family of martingales satisfying all the assumptions of Theorem 3.2. By the procedure presented in [20], we can orthogonalize $\overline{\mathscr{X}} \mathscr{T}$ to achieve an orthogonal family of martingales, the orthogonalized Teugels martingales, possessing the PRP with respect to $\mathbb{F}^{L}$ which fulfils the representation (37) for every $\mathbb{F}^{L}$-adapted, square integrable martingale.

Hermite polynomials. We consider a Lévy process $\left(L, \mathbb{F}^{L}\right)$ with characteristic triplet $\left(\beta, \sigma^{2}, \nu\right)$, where $\nu$ is of the form

$$
\mathrm{d} \nu(x)=h(x) \mathrm{d} x, \quad h(x)>0, \quad x \in \mathbb{R} .
$$

An important class of Lévy processes with Lévy measure as in (40) is, for example, the class of $\alpha$-stable processes (see, e.g., [22, Chap. 3]). We begin with the simpler case of the Cauchy process. A Cauchy process $\left(L, \mathbb{F}^{L}\right)$ is a purely non-Gaussian Lévy process with characteristic triplet $(0,0, \nu)$ and $\mathrm{d} \nu(x):=x^{-2} \mathrm{~d} x$. For a Cauchy process no moment exists and therefore Teugels martingales cannot be introduced. We choose the function $g$ as $g(x):=x \exp \left(-x^{2} / 2\right), x \in \mathbb{R}$, and define $\mathrm{d} \nu^{g}(x):=g^{2}(x) \mathrm{d} \nu(x)=$ $\exp \left(-x^{2}\right) \mathrm{d} x$, which is a finite measure. Let $\left(H_{n}\right)_{n=0,1, \ldots}$ be the sequence of normalized Hermite polynomials. The sequence $\left(H_{n}\right)_{n \geqslant 0}$ is an orthonormal basis for $L^{2}\left(\nu^{g}\right)$. Therefore setting $C_{n}:=g H_{n}, n \geqslant 0$, the family $\mathscr{T}=\left\{C_{n}, n \geqslant 0\right\} \subseteq L^{2}(\nu)$, is a complete orthonormal system in $L^{2}(\nu)$. 
Moreover, each $C_{n}$ is a bounded function. In view of Theorem 4.1 and Theorem 4.3 , the family $\mathscr{X}_{\mathscr{T}}:=\left\{X^{C_{n}}, n \geqslant 0\right\}$ is a family of locally bounded orthogonal martingales with the PRP with respect to $\mathbb{F}^{L}$ and for which (37) holds. Note that the system $\mathscr{T}$ is not stable under multiplication, because the system of Hermite polynomials is not. Therefore, the family $\mathscr{X}_{\mathscr{T}}$ is not compensated-covariation stable. For the general case of a Lévy process with characteristic triplet $\left(\beta, \sigma^{2}, \nu\right)$, where $\nu$ is a Lévy measure as in (40), we introduce the orthogonal basis $\mathscr{T}:=\left\{P_{n}, n \geqslant 0\right\} \subseteq L^{2}(\nu)$ setting $P_{n}:=g H_{n}$, $n \geqslant 0$, where now $g(x):=\left(h(x)^{-1 / 2}\right) \exp \left(-x^{2} / 2\right) \in L^{2}(\nu)$. The associated family of martingales $\mathscr{X}_{\mathscr{T}}:=\left\{\mathrm{W}^{\sigma}\right\} \cup\left\{X^{P_{n}}, n \geqslant 0\right\}$ is orthogonal and possesses the PRP with respect to $\mathbb{F}^{L}$. Therefore (37) holds. In the general case, we cannot expect that $\mathscr{X}_{\mathscr{T}}$ consists of locally bounded martingales. Furthermore, $\mathscr{X}_{\mathscr{T}}$ is not compensated-covariation stable.

Haar wavelet. We consider a Lévy process $\left(L, \mathbb{F}^{L}\right)$ with characteristic triplet $\left(\beta, \sigma^{2}, \nu\right)$ and Lévy measure as in (40). Let $\lambda$ be the Lebesgue measure on $\mathbb{R}$, and let $\psi \in L^{2}(\lambda)$. If the system $\left\{\psi_{j k}: \psi_{j k}(x)=2^{\frac{j}{2}} \psi\left(2^{j} x-k\right), x \in\right.$ $\mathbb{R}, j, k \in \mathbb{Z}\}$ of functions is a complete orthonormal system in $L^{2}(\lambda)$, then the function $\psi$ is called a wavelet. An example of a wavelet, known as Haar wavelet, is the function defined by $\psi(x):=1$, if $x \in[0,1 / 2)$; $\psi(x):=-1$ if $x \in[1 / 2,1)$, and $\psi(x):=0$ otherwise. The system $\widetilde{T}:=\left\{\psi_{j k}, j, k \in \mathbb{Z}\right\}$ generated by $\psi$ is the Haar basis (cf. [25, Chap. 1]). The system $\mathscr{T}:=\left\{h^{-1 / 2} \psi_{j k}, j, k \in \mathbb{Z}\right\}$ is a complete orthogonal system in $L^{2}(\nu)$. From Theorem 4.3, $\mathscr{X}_{\mathscr{T}}:=\left\{\mathrm{W}^{\sigma}\right\} \cup\left\{X^{f}, f \in \mathscr{T}\right\}$ is a system of orthogonal martingales and possesses the PRP with respect to $\mathbb{F}^{L}$. Therefore (4.3) holds. We stress that $\mathscr{X}_{\mathscr{T}}$ is not a compensated-covariation stable family, because $\mathscr{T}$ is not stable under multiplication. Furthermore, in general, we cannot expect that $\mathscr{T}$ is a system of bounded functions.

Appendix A: Denseness of polynomials. Let $\mathscr{Z}$ be an arbitrary family of (real-valued) random variables. By $\mathscr{F}^{\mathscr{Z}}$ we denote the $\sigma$-algebra generated by $\mathscr{Z}$. We consider the probability space $\left(\Omega, \mathscr{F}^{\mathscr{Z}}, \mathbb{P}\right)$ and the Hilbert space $L^{2}\left(\Omega, \mathscr{F}^{\mathscr{Z}}, \mathbb{P}\right)$. By $\mathscr{K}$ we denote the system

$$
\mathscr{K}:=\left\{\prod_{i=1}^{m} Z_{i}, Z_{1}, \ldots, Z_{m} \in \mathscr{Z}, m \in \mathbb{N} \cup\{0\}\right\}
$$

of monomials in $Z \in \mathscr{Z}$.

In this appendix we show that, under the assumption that for every $Z \in \mathscr{Z}$ there is a finite exponential moment of arbitrary small order, the set $\mathscr{K}$ of monomials is a total system (i.e., its linear hull is dense) in $L^{2}\left(\Omega, \mathscr{F}^{\mathscr{Z}}, \mathbb{P}\right)$. Although this result has to be considered as part of the mathematical folklore, we were not able to find an adequate reference easily accessible by the reader. For convenience, we therefore decided to present 
here a quite short and elementary direct proof of this fact, without using tools from complex function theory.

We start with a preliminary lemma. We consider a finite measure space $(E, \mathscr{E}, \mu)$ and we denote by $\mathbb{B}=\mathbb{B}(E, \mathbb{R})$ the space of bounded measurable (real) functions on $(E, \mathscr{E})$. By $L^{q}(\mu)$ we denote the space of measurable $q$ integrable functions on $(E, \mathscr{E}, \mu)$. We use the notation $\mu(f):=\int_{E} f(x) \mathrm{d} \mu(x)$ if the integral on the right-hand side exists. We recall that if $\mathscr{C}$ is a class of real functions, by $\operatorname{Span}(\mathscr{C})$ we denote the linear hull of $\mathscr{C}$.

Lemma A.1. Let $\mathscr{T} \subseteq L^{q}(\mu)$ be a subset of $\mathbb{B}$. Then $\mathscr{T}$ is total in $L^{q}(\mu)$ if: (i) $\mathscr{T}$ is stable under multiplication; (ii) $\sigma(\mathscr{T})=\mathscr{E}$; (iii) $1=1_{E} \in \mathscr{T}$.

P r o o f. Note that $\mathbb{B} \subseteq L^{q}(\mu)$. We denote by $\mathscr{H}$ the closure of $\operatorname{Span}(\mathscr{T})$ in $L^{q}(\mu)$ and put $\mathscr{D}:=\mathscr{H} \cap \mathbb{B}$. Clearly, $\mathscr{D}$ is a linear space and $\mathscr{T} \subseteq \mathscr{D}$. By assumption, $1 \in \mathscr{T}$ and hence $1 \in \mathscr{D}$. Moreover, $\mathscr{D}$ is closed under monotone convergence of uniformly bounded nonnegative functions, as a consequence of the finiteness of $\mu$ and of Lebesgue's dominated convergence theorem. Consequently, $\mathscr{D}$ is a monotone vector space and by the monotone class theorem for systems of functions (cf. [23, Appendix A0]) we get $\mathscr{D}=\mathbb{B}$ which yields $\mathbb{B} \subseteq \mathscr{H}$ and hence $L^{q}(\mu)=\mathscr{H}$ because $\mathscr{H}$ is closed and $\mathbb{B}$ is dense in $L^{q}(\mu)$. Lemma $\mathrm{A} .1$ is proved.

Now we come back to the problem of the totality of $\mathscr{K}$ in $L^{2}\left(\Omega, \mathscr{F}^{\mathscr{Z}}, \mathbb{P}\right)$. We require the following property for the family $\mathscr{Z}$.

Assumption A.2. For every $Z \in \mathscr{Z}$ there exists a positive constant $c$, which may depend on $Z$, such that $\mathbb{E}[\exp (c|Z|)]<+\infty$.

By $\mathscr{C}$ we denote the linear hull of the cosine of linear combinations of elements of $\mathscr{Z}$, that is,

$$
\mathscr{C}:=\operatorname{Span}\left(\left\{\cos \left(\sum_{i=1}^{m} u_{i} Z_{i}\right), u_{i} \in \mathbb{R}, Z_{i} \in \mathscr{Z}, i=1, \ldots, m ; m \in \mathbb{N}\right\}\right) .
$$

Lemma A.2. The system $\mathscr{C}$ introduced in (A.2) is dense in $L^{2}\left(\Omega, \mathscr{F}^{\mathscr{Z}}, \mathbb{P}\right)$.

P r o o f. Clearly, $\mathscr{C}$ is a linear system of bounded functions containing $1=1_{\Omega}$. Moreover, $\mathscr{C}$ is stable under multiplication in view of $\cos (x) \cos (y)=$ $(\cos (x+y)+\cos (x-y)) / 2$ for each $x, y \in \mathbb{R}$, by the angle addition theorem for the cosine function. To apply Lemma A.1 and conclude that $\mathscr{C}$ is dense in $L^{2}\left(\Omega, \mathscr{F}^{\mathscr{Z}}, \mathbb{P}\right)$, we only need to show that $\mathscr{C}$ generates $\mathscr{F}^{\mathscr{Z}}$. The system of functions $\{\cos (u x), u \in \mathbb{R}\}$ is separating points. Let $\mathscr{E}_{0}$ be the $\sigma$-algebra generated by $\{\cos (u x), u \in \mathbb{R}\}$. Then $\mathscr{E}_{0}$ is separable. The space $(\mathbb{R}, \mathscr{B}(\mathbb{R}))$ is a Blackwell space (cf. [6, Definition 3.24]) and by Blackwell's theorem (cf. $\left[6\right.$, Theorem 3.26]) we obtain $\mathscr{E}_{0}=\mathscr{B}(\mathbb{R})$. This implies that $\sigma(\mathscr{C})=\mathscr{F}^{\mathscr{Z}}$ and the proof of the claim is finished. Lemma A.2 is proved. 
Now we are ready to state and prove the following theorem.

Theorem A.1. Let $\mathscr{Z}$ be an arbitrary family of random variables satisfying Assumption A.2. Then the system $\mathscr{K}$ of monomials defined by (A.1) is total in $L^{2}\left(\Omega, \mathscr{F}^{\mathscr{Z}}, \mathbb{P}\right)$.

P r o o f. Using the inequality between geometric and arithmetic means (or the convexity of the exponential function) it can easily be seen that $\operatorname{Span}(\mathscr{Z})$ again satisfies Assumption A.2. If we denote by $\mathscr{K}_{1}$ the set of monomials defined by (A.1) with respect to $\operatorname{Span}(\mathscr{Z})$ it follows $\operatorname{Span}\left(\mathscr{K}_{1}\right)=$ $\operatorname{Span}(\mathscr{K})$. Hence the theorem is proved if we show that $\operatorname{Span}\left(\mathscr{K}_{1}\right)$ is dense in $L^{2}\left(\Omega, \mathscr{F}^{\mathscr{Z}}, \mathbb{P}\right)$. Therefore, without loss of generality we can assume that $\mathscr{Z}$ is a linear space: If this is not satisfied we can replace $\mathscr{Z}$ by the family $\operatorname{Span}(\mathscr{Z})$.

From now on we assume that $\mathscr{Z}$ is a linear space. So $\mathscr{C}:=$ $\operatorname{Span}(\{\cos (Z), Z \in \mathscr{Z}\})$. Let $\mathscr{P}$ be the linear hull of $\mathscr{K}$, where $\mathscr{K}$ has been introduced in (A.1), and let $\overline{\mathscr{P}}$ be the closure of $\mathscr{P}$ in $L^{2}(\Omega, \mathscr{F} \mathscr{Z}, \mathbb{P})$. To prove the assertion of the theorem, in view of Lemma A.2, it is sufficient to show that $\mathscr{C} \subseteq \overline{\mathscr{P}}$.

For this aim, let $Z \in \mathscr{Z}$ be arbitrary but fixed and let $c>0$ be chosen such that $\mathbb{E}[\exp (c|Z|)]<+\infty$. By induction over $k$ we shall prove that $Z^{l} \cos (u Z)$ belongs to $\mathscr{P}$ for every $u$ with $|u| \leqslant k c / 4$ and for every $l \in \mathbb{N} \cup\{0\}$. We assume that $u \geqslant 0$, the case $u<0$ is treated analogously.

To begin with, we consider the case $k=1$. Note that, because of the series expansion of the cosine function, we have

$$
\lim _{m \rightarrow+\infty} \sum_{n=0}^{m}(-1)^{n} \frac{(u Z)^{2 n}}{(2 n) !}=\cos (u Z)
$$

pointwise. On the other side, for $0 \leqslant u \leqslant c / 4$, we have

$$
\begin{gathered}
\left(Z^{l} \cos (u Z)-Z^{l} \sum_{n=0}^{m}(-1)^{n} \frac{(u Z)^{2 n}}{(2 n) !}\right)^{2}=Z^{2 l}\left(\sum_{n=m+1}^{\infty}(-1)^{n} \frac{(u Z)^{2 n}}{(2 n) !}\right)^{2} \\
\leqslant Z^{2 l}\left(\sum_{n=m+1}^{\infty} \frac{|c / 4|^{2 n}|Z|^{2 n}}{(2 n) !}\right)^{2}=Z^{2 l} \mathrm{e}^{c|Z| / 2}
\end{gathered}
$$

By Hölder's inequality we get $\mathbb{E}\left[Z^{2 l} \mathrm{e}^{c|Z| / 2}\right] \leqslant\left(\mathbb{E}\left[Z^{4 l}\right]\right)^{1 / 2}\left(\mathbb{E}\left[\mathrm{e}^{c|Z|}\right]\right)^{1 / 2}<+\infty$. Using Lebesgue's dominated convergence theorem, we deduce that the polynomials $Z^{l} \sum_{n=0}^{m}(-1)^{n}(u Z)^{2 n} /(2 n)$ ! converge in $L^{2}\left(\Omega, \mathscr{F}^{\mathscr{Z}}, \mathbb{P}\right)$ to $Z^{l} \cos (u Z)$ and therefore $Z^{l} \cos (u Z) \in \overline{\mathscr{P}}$ for all $0 \leqslant u \leqslant c / 4$ and $l \in \mathbb{N} \cup\{0\}$.

Now we assume that $Z^{l} \cos (u Z)$ belongs to $\overline{\mathscr{P}}$ for all $0 \leqslant u \leqslant k c / 4$ and $l \in \mathbb{N} \cup\{0\}$ and prove that $Z^{l} \cos (u Z)$ belongs to $\overline{\mathscr{P}}$ for all $0 \leqslant u \leqslant(k+1) c / 4$ and $l \in \mathbb{N} \cup\{0\}$. For this we choose $u \in\left(v_{k}, v_{k+1}\right]$, where $v_{j}:=j c / 4$, $j \in \mathbb{N}$. Defining $v:=u-v_{k}$, we obtain the relation $Z^{l} \cos (v Z) \cos \left(v_{k} Z\right)=$ 
$Z^{l}\left(\cos \left(\left(v+v_{k}\right) Z\right)+\cos \left(\left(v-v_{k}\right) Z\right)\right) / 2$, from which we can easily deduce the identity

$$
Z^{l} \cos (u Z)=2 Z^{l} \cos (v Z) \cos \left(v_{k} Z\right)-Z^{l} \cos \left(\left(v-v_{k}\right) Z\right) .
$$

Obviously, $-v_{k} \leqslant v-v_{k} \leqslant 0$, hence $\left|v-v_{k}\right| \leqslant v_{k}$. By the induction hypothesis and from this follows that $Z^{l} \cos \left(\left(v-v_{k}\right) Z\right)$ belongs to $\overline{\mathscr{P}}$. Thus, to verify that $Z^{l} \cos (u Z) \in \overline{\mathscr{P}}$, it is sufficient to prove that $Z^{l} \cos (v Z) \cos \left(v_{k} Z\right)$ belongs to $\overline{\mathscr{P}}$. Note that we have the pointwise convergence

$$
Z^{l} \cos (v Z) \cos \left(v_{k} Z\right)=\lim _{m \rightarrow \infty} \sum_{n=0}^{m}(-1)^{n} \frac{(v Z)^{2 n}}{(2 n) !} Z^{l} \cos \left(v_{k} Z\right) .
$$

Using the induction hypothesis, we obtain $Z^{2 n+l} \cos \left(v_{k} Z\right) \in \overline{\mathscr{P}}$, from which follows that

$$
\sum_{n=0}^{m}(-1)^{n} \frac{(v Z)^{2 n}}{(2 n) !} Z^{l} \cos \left(v_{k} Z\right) \quad \text { belongs to } \overline{\mathscr{P}} \text {. }
$$

It remains to show the $L^{2}$-convergence of this series to $Z^{l} \cos (v Z) \cos \left(v_{k} Z\right)$. For this we estimate

$$
\begin{aligned}
\left(Z^{l} \cos (v Z)\right. & \left.\cos \left(v_{k} Z\right)-\sum_{n=0}^{m}(-1)^{n} \frac{(v Z)^{2 n}}{(2 n) !} Z^{l} \cos \left(v_{k} Z\right)\right)^{2} \\
& \leqslant\left(\sum_{n=m+1}^{\infty} \frac{(v Z)^{2 n}}{(2 n) !}\right)^{2} Z^{2 l}\left(\cos \left(v_{k} Z\right)\right)^{2} \leqslant Z^{2 l} \mathrm{e}^{c|Z| / 2}
\end{aligned}
$$

and $\mathbb{E}\left[Z^{2 l} \mathrm{e}^{c|Z| / 2}\right] \leqslant\left(\mathbb{E}\left[Z^{4 l}\right]\right)^{1 / 2}\left(\mathbb{E}\left[\mathrm{e}^{c|Z|}\right]\right)^{1 / 2}<+\infty$. Therefore we can apply Lebesgue's dominated convergence theorem and obtain $Z^{l} \cos (v Z) \cos \left(v_{k} Z\right) \in$ $\overline{\mathscr{P}}$. This completes the proof by induction. Setting $l=0$ and $u=1$ we can now conclude that $\cos (Z)$ belongs to $\overline{\mathscr{P}}$ for all $Z \in \mathscr{Z}$ which proves the theorem.

\section{REFERENCES}

1. Boel R., Varaiya P., Wong E. Martingales on jump processes. I: Representation results. - SIAM J. Control Optim., 1975, v. 13, № 5, p. 999-1021.

2. Clark J. M. C. The representation of functionals of Brownian motion by stochastic integrals. - Ann. Math. Statist., 1970, v. 41, № 4, p. 1282-1295.

3. Chou C.S., Meyer P.-A. Sur la répresentation des martingales comme intégrales stochastique dans les processus ponctuels. - Lecture Notes in Math., 1975, v. 465, p. $226-236$.

4. Davis M.A.H., Varaiya P. The multiplicity of an increasing family of $\sigma$-fields. Ann. Probab., 1974, v. 2, № 5, p. 958-963.

5. Davis M. A.H. The representation of martingales of jump processes. - SIAM J. Control Optim., 1976, v. 14, № 4, p. 623-638. 
6. Dellacherie C., Meyer P.-A. Probabilities and Potential. Amsterdam-New York: North-Holland, 1978, 189 p. (North-Holland Math. Stud., v. 29.)

7. Elliott R. J. Stochastic integrals for martingales of a jump process with partially accessible jump times. - Z. Wahrscheinlichkeitstheor. verw. Geb., 1976, v. 36, № 3, p. 213-266.

8. Engelbert H. J., Hess J. Stochastic integrals of continuous local martingales. I, II. Math. Nachr., 1980, v. 97, p. 325-343; 1981, v. 100, p. 249-269.

9. Гальчук Л. И. Представление некоторых мартингалов. - Теория вероятн. и ее примен., 1976, т. 21 , в. 3, с. 613-620.

10. Гихман И.И., Скороход А.В. Теория случайных процессов, т. 1. М.: Наука, $1971,664 \mathrm{c}$.

11. Itô K. Multiple Wiener integral. — J. Math. Soc. Japan, 1951, v. 3, № 1, p. 157-169.

12. Itô $K$. Spectral type of the shift transformation of differential processes with stationary increments. - Trans. Amer. Math. Soc., 1956, v. 81, p. 253-263.

13. Jacod J. Calcul stochastique et problèmes de martingales. - Lecture Notes in Math., 1979, v. 714 , p. $1-539$.

14. Jacod J., Shiryaev A. Limit Theorems for Stochastic Processes. Berlin: SpringerVerlag, 2003, 661 p. (Grundlehren Math. Wiss., v. 288.)

15. Jacod J., Yor M. Étude des solutions extrémales et représentation intégrale des solutions pour certains problèmes de martingales. - Z . Wahrscheinlichkeitstheor. verw. Geb., 1977, v. 38, p. 83-125.

16. Кабанов Ю. М. Представление функционалов от винеровского и пуассоновского процессов в виде стохастических интегралов. - Теория вероятн. и ее примен., 1973 , т. 18 , в. 2 , с. $376-380$.

17. Кабанов Ю. М. Интегральные представления функционалов от процессов с независимыми приращениями. - Теория вероятн. и ее примен., 1974, т. 19, в. 4, c. 889-893.

18. Kallenberg O. Foundations of Modern Probability. New York: Springer-Verlag, 2002, $638 \mathrm{p}$.

19. Kunita $H$. Representation of martingales with jumps and applications to finance. Stochastic Analysis and Related Topics. Tokyo: Math. Soc. Japan, 2004, p. 209232. (Adv. Stud. Pure Math., v. 41.)

20. Kunita H., Watanabe S. On square integrable martingales. - Nagoya Math. J., 1967 , v. 30, p. 209-245.

21. Nualart D., Schoutens $W$. Chaotic and predictable representations for Lévy processes. - Stochastic Process. Appl., 2000, v. 90, № 1, p. 109-122.

22. Sato K. Lévy Processes and Infinitely Divisible Distributions. Cambridge: Cambridge Univ. Press, 1999, 521 p. (Cambridge Stud. Adv. Math., v. 68.)

23. Sharpe M. General Theory of Markov Processes. Boston: Academic Press, 1988, 419 p. (Pure Appl. Math., v. 133.)

24. Wang J. Some remarks on processes with independent increments. - Lecture Notes in Math., 1981, v. 850, p. 627-631.

25. Wojtaszczyk P. A Mathematical Introduction to Wavelets. Cambridge: Cambridge Univ. Press, 1997, 261 p. (London Math. Soc. Stud. Texts, v. 37.)

Поступила в редакцию

22.VII.2013

Исправленный вариант

22.II. 2014 